\title{
Into the amplituhedron
}

\author{
Nima Arkani-Hamed ${ }^{a}$ and Jaroslav Trnka ${ }^{b}$ \\ ${ }^{a}$ School of Natural Sciences, Institute for Advanced Study, \\ Princeton, NJ 08540, U.S.A. \\ ${ }^{b}$ Walter Burke Institute for Theoretical Physics, California Institute of Technology, \\ Pasadena, CA 91125, U.S.A. \\ E-mail: arkani@ias.edu, trnka@caltech.edu
}

ABSTRACT: We initiate an exploration of the physics and geometry of the amplituhedron, starting with the simplest case of the integrand for four-particle scattering in planar $\mathcal{N}=4$ SYM. We show how the textbook structure of the unitarity double-cut follows from the positive geometry. We also use the geometry to expose the behavior of the multicollinear limit, providing a direct motivation for studying the logarithm of the amplitude. In addition to computing the two and three-loop integrands, we explore various lower-dimensional faces of the amplituhedron, thereby computing non-trivial cuts of the integrand to all loop orders.

KEYWords: Supersymmetric gauge theory, Scattering Amplitudes

ARXIV EPRINT: 1312.7878 


\section{Contents}

1 Geometry and physics of the amplituhedron 1

2 One loop geometry 4

3 Warmup exercises $\quad 6$

$\begin{array}{lll}4 & \text { Two loops } & 10\end{array}$

5 Generalities on cuts 12

6 Unitarity from positivity 14

$\begin{array}{lll}7 & \text { Three loops } & 16\end{array}$

$\begin{array}{llr}8 & \text { Multi-collinear region } & 18\end{array}$

$\begin{array}{llr}9 & \text { Log of the amplitude } & 19\end{array}$

10 Some faces of the amplituhedron $\quad 23$

$\begin{array}{ll}11 \text { Four particle outlook } & 28\end{array}$

\section{Geometry and physics of the amplituhedron}

In [1], we introduced a new geometric object - the Amplituhedron - underlying the physics of scattering amplitudes for $\mathcal{N}=4 \mathrm{SYM}$ in the planar limit. At tree level, the amplituhedron is a natural generalization of "the inside of a convex polygon". Loops arise by extending the geometry to incorporate the idea of "hiding particles" in the only natural way possible.

The amplituhedron $\mathcal{A}_{n, k, L}$ for $n$-particle $\mathrm{N}^{k} \mathrm{MHV}$ amplitudes at $L$ loops, lives in $G(k, k+4 ; L)$, which is the space of $k$-planes $Y$ in $k+4$ dimensions, together with $L$ 2-planes $\mathcal{L}_{1}, \cdots, \mathcal{L}_{L}$ in the 4 dimensional complement of $Y$. The external data are given by a collection of $n(k+4)$ dimensional vectors $Z_{a}^{I}$. Here $a=1, \cdots n$, and $I=1, \cdots,(k+4)$. This data is taken to be "positive", in the sense that all the ordered $(k+4) \times(k+4)$ determinants $\left\langle Z_{a_{1}} \cdots Z_{a_{k+4}}\right\rangle>0$ for $a_{1}<\cdots<a_{k+4}$. The subspace of $\mathcal{A}_{n, k, L}$ of $G(k, k+4 ; L)$ is determined by a "positive" linear combination of the (positive) external data. The $k$-plane is $Y_{\alpha}^{I}$, and the 2-planes are $\mathcal{L}_{\gamma(i)}^{I}$, where $\gamma=1,2$ and $i=1, \ldots, L$. The amplituhedron is the space of all $Y, \mathcal{L}_{(i)}$ of the form

$$
Y_{\alpha}^{I}=C_{\alpha a} Z_{a}^{I}, \quad \mathcal{L}_{\gamma(i)}^{I}=D_{\gamma a(i)} Z_{a}^{I}
$$


where the $C_{\alpha a}$ specifies a $k$-plane in $n$-dimensions, and the $D_{\gamma a(i)}$ are $L$ 2-planes living in the $(n-k)$ dimensional complement of $C$, with the positivity property that for any $0 \leq l \leq L$, all the ordered maximal minors of the $(k+2 l) \times n$ matrix

$$
\left(\begin{array}{c}
D_{\left(i_{1}\right)} \\
-\vdots \\
\hdashline D_{\left(i_{l}\right)} \\
\hline C
\end{array}\right)
$$

are positive.

There is a canonical rational form $\Omega_{n, k ; L}$ associated with $\mathcal{A}_{n, k ; L}$, with the property of having logarithmic singularities on all the lower-dimensional boundaries of $\mathcal{A}_{n, k ; L}$. The loop integrand form for the super-amplitude is naturally extracted from $\Omega_{n, k ; L}$ [1].

The amplituhedron can be defined in a few lines, as we have just done. But the resulting geometry is incredibly rich and intricate - as it must be, to generate all the structure found in planar $\mathcal{N}=4 \mathrm{SYM}$ scattering amplitudes to all loop orders! For instance, the singularity structure of the amplitude is reflected in the geometry of the various boundaries of the amplituhedron; studying this geometry in some of the simplest cases allows us to see the emergence of locality and unitarity from positive geometry.

Even just the tree amplituhedron generalizes the positive Grassmannian $G_{+}(k, n)$ [2]. A complete understanding of $G_{+}(k, n)$ revealed many surprising connections to other structures, from the fundamentally combinatorial backbone of affine permutations, to cluster algebras, to the physical connection with on-shell processes [2-5]. It is natural to expect the full amplituhedron $\mathcal{A}_{n, k, L}$ to have a much richer structure. A complete understanding of the full geometry of the amplituhedron, at the same level as our understanding of the positive Grassmannian, will likely involve further physical and mathematical ideas. Our goal in this note is to begin laying the groundwork for this exploration, by looking at various simple aspects of amplituhedron geometry in the simplest non-trivial case of clear physical interest.

While the tree amplituhedron generalizes the positive Grassmannian in a direct way, extending the notion of positivity to external data, the extension of positivity associated with "hiding particles" which gives rise to loops is more novel and interesting. The very simplest case of four-particle scattering has $k=0, n=4$. Here, we don't have the additional structure of Grassmann components for the external data [1], the external data are just the ordinary bosonic momentum-twistor [6] variables $Z_{1}^{I}, Z_{2}^{I}, Z_{3}^{I}, Z_{4}^{I}$, for $I=1, \cdots, 4$. Furthermore, the constraint of positivity for external data is trivial in this case; indeed using a $G L(4)$ transformation we can set the $4 \times 4$ matrix $\left(Z_{1}, \cdots, Z_{4}\right)$ to identity. The loop variables are just lines in momentum-twistor space (or better, two-planes in four-dimensions), which correspond to points in the (dual) space-time. Having set the $Z$ matrix to the identity, each $2 \times 4$ matrix for the lines $\mathcal{L}_{\gamma(a)}^{I}$ is simply identified with the $D$ matrices $D_{(i)}$.

The amplituhedron positivity constraints are that the all the ordered minors of each $D_{(i)}$ matrix are positive

$$
(12)_{i},(13)_{i},(14)_{i},(23)_{i},(24)_{i},(34)_{i}>0
$$


We also have mutual positivity, that the $4 \times 4$ determinant $\left\langle D_{(i)} D_{(j)}\right\rangle>0$, which tells us that

$$
(12)_{i}(34)_{j}+(23)_{i}(14)_{j}+(34)_{i}(12)_{j}+(14)_{i}(23)_{j}-(13)_{i}(24)_{j}-(24)_{i}(13)_{j}>0
$$

We can also express these conditions in a convenient gauge, where

$$
D_{(i)}=\left(\begin{array}{cccc}
1 & x_{i} & 0 & -w_{i} \\
0 & y_{i} & 1 & z_{i}
\end{array}\right)
$$

Then the positivity of each $D_{(i)}$ simply tells us that

$$
x_{i}, y_{i}, z_{i}, w_{i}>0
$$

while the mutual positivity conditions become

$$
\left(x_{i}-x_{j}\right)\left(z_{i}-z_{j}\right)+\left(y_{i}-y_{j}\right)\left(w_{i}-w_{j}\right)<0
$$

In this note we study various aspects of the geometry defined by these inequalities, as well as the corresponding canonical form $\Omega$, which directly gives us the loop integrand for four-particle scattering. Of course the four-particle amplitude has been an object of intensive study for many years [7-11], with loop integrand now available through seven loops. But our approach will be fundamentally different from previous works. We will not begin by drawing planar diagrams made out of "boxes", we will make no mention of recursion relations, we will not make ansaetze for the integrand which are checked against cuts, and we will make no mention of physical constraints from exponentiation of infrared divergences etc. Instead, we will discover all the known general properties of the loop integrand, and many other properties besides, directly by studying the positive geometry of the amplituhedron.

We will start with a lightning review of the one-loop geometry, which is just that of $G_{+}(2,4)$, mostly to define some notation and nomenclature. We then do some warm-up exercises for associating canonical forms $\Omega$ with spaces specified by particularly simple inequalities, which will come in handy in later sections. The first non-trivial case with mutual positivity is obviously two loops, and we show how to triangulate the space and extract the loop integrand, matching the well-known result given as a sum of two doubleboxes. Interestingly, while our triangulation of the two-loop amplituhedron is manifestly "positive", the sum of double-boxes is not, with each term having singularities outside the amplituhedron that only cancel in the sum.

We then make some general observation on the structure of certain cuts of the amplitude, which correspond to various boundaries of the amplituhedron. In particular, the textbook understanding of unitarity as following from the break-up of the loop integrand into two parts sewed together on the "unitarity cut" follows in a beautiful way from positive geometry. These general results and some further explicit triangulations also allow us to determine the three-loop integrand. We move on to exploring another natural set of cuts that take the amplitude into the multi-collinear region. This exposes a fascinating property of cuts of the multi-loop integrand: the residues depend not only on the final cut 
geometry, but also on the path taken to reach that geometry. Studying the combinatorics of this path dependence naturally motivates looking at the logarithm of the amplitude, and explains why the log has such good IR behavior.

From our new perspective, the determination of the integrand to all loop orders requires a complete understanding of the full amplituhedron geometry. We have not yet achieved this yet, but we believe that a systematic approach to this problem is possible. As a prelude, we give a survey of some of the lower-dimensional "faces" of the amplituhedron. We can explicitly triangulate these faces and find their corresponding canonical forms, which give us cuts of the full integrand. This already gives us highly non-trivial all-loop order information about the integrand, in many cases not readily available from any other approach.

\section{One loop geometry}

At one loop we have a single line $\mathcal{L}_{1} \mathcal{L}_{2}$, which we often also called " $(A B)$ ". The geometry is given by the positive Grassmannian $G_{+}(2,4)$. The external data form a polygon in $\mathbb{P}^{3}$ with vertices $Z_{1}, Z_{2}, Z_{3}, Z_{4}$ and edges $Z_{1} Z_{2}, Z_{2} Z_{3}, Z_{3} Z_{4}, Z_{1} Z_{4}$.

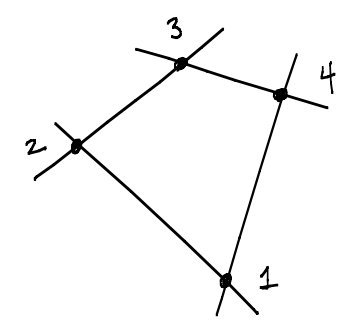

The line $A B=\mathcal{L}_{1} \mathcal{L}_{2}$ is parametrized as

$$
\mathcal{L}_{\gamma}^{I}=D_{\gamma a} Z_{a}^{I}
$$

where $\gamma=1,2$ and $a, I=1, \ldots, 4$. The matrix $D$ represents a cell of the positive Grassmannian $G_{+}(2,4)$, in the generic case it is a top cell. In one particularly convenient gauge-fixing we can write

$$
D=\left(\begin{array}{cccc}
1 & x & 0 & -w \\
0 & y & 1 & z
\end{array}\right)
$$

where $x, y, z, w>0$. This gauge-fixing of the $D$ matrix covers all boundaries by sending variables $x, y, z, w$ to zero or infinity.

The form with logarithmic singularities on the boundaries of the space is trivially

$$
\Omega=\frac{d x}{x} \frac{d y}{y} \frac{d w}{w} \frac{d z}{z}
$$

The boundaries occur when one of the variables approaches 0 or $\infty$. We can easily translate this expression back to momentum twistor space by solving two linear equations:

$$
Z_{A}=Z_{1}+x Z_{2}-w Z_{4}, \quad Z_{B}=y Z_{2}+Z_{3}+z Z_{4}
$$


which gives

$$
\Omega=\frac{\left\langle A B d^{2} Z_{A}\right\rangle\left\langle A B d^{2} Z_{B}\right\rangle\langle 1234\rangle^{2}}{\langle A B 12\rangle\langle A B 23\rangle\langle A B 34\rangle\langle A B 14\rangle}
$$

We now describe the boundaries of this space in detail - these are nothing but all the cells of $G_{+}(2,4)$, which have also been described at length in e.g. [4, 5]. We describe them in detail here since the same geometry will arise repeatedly in the context of cuts of the multiloop amplitudes. At the level of the form they correspond to logarithmic singularities. In giving co-ordinates for the boundaries, we will freely use different gaugefixings as convenient for any given case, with all parameters positive. They will always be trivially related to boundaries of (2.2).

The first type of boundary occurs when line $A B$ intersects one of the lines $Z_{1} Z_{2}, Z_{2} Z_{3}$, $Z_{3} Z_{4}$ or $Z_{1} Z_{4}$. In the gauge-fixing (2.2) this sets one of the variables to $x, y, z, w$ to 0 . In particular,

$$
\langle A B 12\rangle=w, \quad\langle A B 23\rangle=z, \quad\langle A B 34\rangle=y, \quad\langle A B 14\rangle=x
$$

where we suppressed $\langle 1234\rangle$. For cutting $Z_{1} Z_{2},\langle A B 12\rangle=w=0$ we get

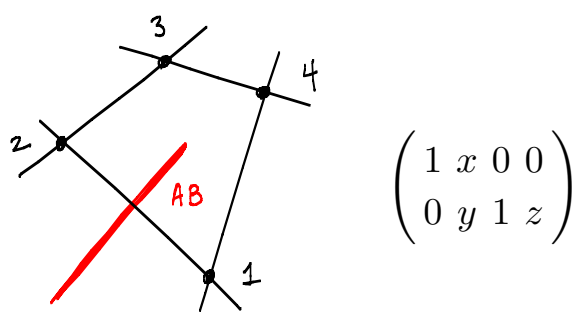

In all four cases the form is the dlog of the remaining three variables; we will suppress writing it explicitly.

The second type of boundary occurs when the line $A B$ intersects two lines $Z_{i} Z_{i+1}$ and $Z_{j} Z_{j+1}$. There are two distinct cases. If we cut two non-adjacent lines $Z_{1} Z_{2}, Z_{3} Z_{4}$ or $Z_{2} Z_{3}, Z_{1} Z_{4}$ there is just one solution. For the first one $\langle A B 12\rangle=\langle A B 34\rangle=0$ we have

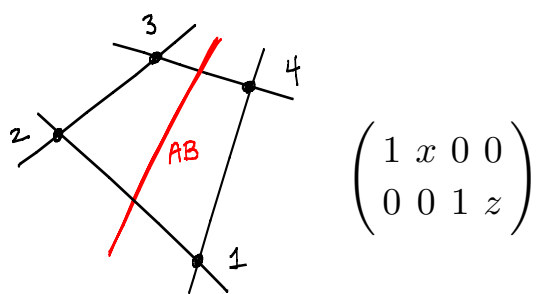

In the second case we intersect two adjacent lines. Let us cut $Z_{1} Z_{2}, Z_{2} Z_{3}$ (the other three cases are cyclically related), ie. $\langle A B 12\rangle=\langle A B 23\rangle=0$. There are two different solutions 
- either the line $A B$ passes through $Z_{2}$ or the line $A B$ lies in the plane $\left(Z_{1} Z_{2} Z_{3}\right)$.

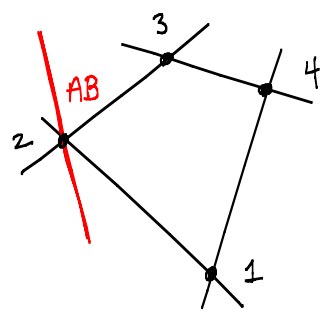

$$
\left(\begin{array}{llll}
1 & 0 & 0 & 0 \\
0 & y & 1 & z
\end{array}\right)
$$

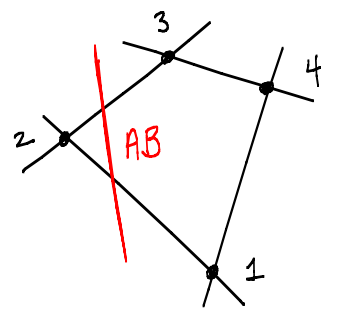

$$
\left(\begin{array}{llll}
1 & x & 0 & 0 \\
0 & y & 1 & 0
\end{array}\right)
$$

There are two different type-three boundaries. The first type is a triple cut - the line $A B$ intersects three of four lines. One representative is $\langle A B 12\rangle=\langle A B 23\rangle=\langle A B 34\rangle=0$. There are two solutions to this problem. Either $A B$ passes through $Z_{2}$ and intersects the line $Z_{3} Z_{4}$ or $A B$ passes through $Z_{3}$ and intersects the line $Z_{1} Z_{2}$.

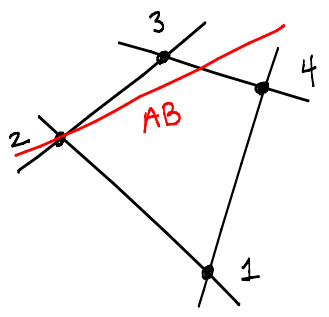

$$
\left(\begin{array}{llll}
0 & 1 & 0 & 0 \\
0 & 0 & 1 & \alpha
\end{array}\right)
$$

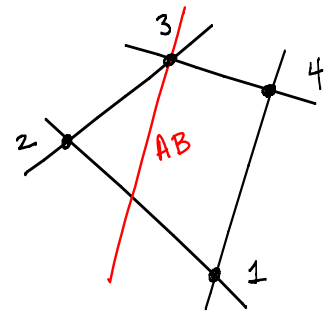

$$
\left(\begin{array}{cccc}
0 & 0 & 1 & 0 \\
-\alpha & -1 & 0 & 0
\end{array}\right)
$$

There is also a "composite" cut when we cut only two lines while imposing three constraints. We can pass $A B$ through $Z_{2}$ while lying in the plane plane $\left(Z_{1} Z_{2} Z_{3}\right)$.

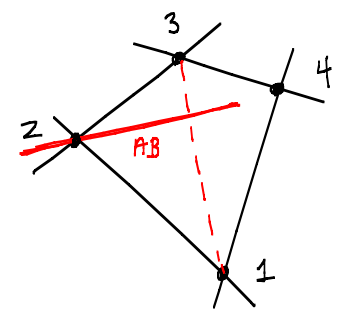

$$
\left(\begin{array}{cccc}
0 & 1 & 0 & 0 \\
-\alpha & 0 & 1 & 0
\end{array}\right)
$$

Finally, for the quadruple cuts we can either cut all four lines which localizes $A B$ to $A B=$ $Z_{1} Z_{3}$ or $A B=Z_{2} Z_{4}$, or we can consider the "composite" cut $A B=Z_{1} Z_{2}$ (and cyclically related) which cuts only three lines (not $Z_{3} Z_{4}$ ) while still imposing four constraints.

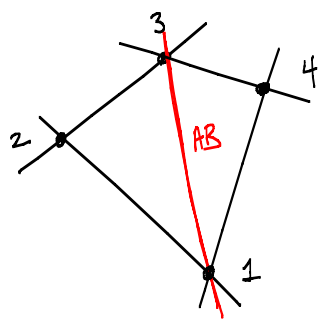

$$
\left(\begin{array}{llll}
1 & 0 & 0 & 0 \\
0 & 0 & 1 & 0
\end{array}\right)
$$

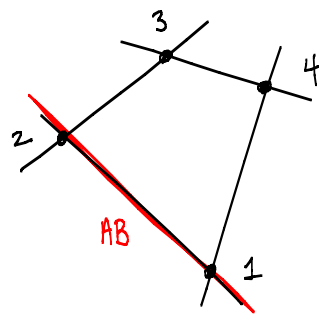

$$
\left(\begin{array}{llll}
1 & 0 & 0 & 0 \\
0 & 1 & 0 & 0
\end{array}\right)
$$

\section{Warmup exercises}

The amplituhedron is defined by various positivity conditions. We will shortly be "triangulating" the spaces defined by these inequalities and finding the canonical form $\Omega$ associated 
with them. But it will be helpful to practice on some simpler cases, which will also later be useful to determining amplitudes and cuts of amplitudes.

Let us start with a trivial example; suppose we have

$$
a<x<b
$$

It's obvious that the form is $\frac{1}{x-a}-\frac{1}{x-b}$, but lets reproduce this in a heavy-handed way, from the viewpoint of using "positive co-ordinates". In this case, we can write

$$
x=a+(b-a) \frac{\alpha}{1+\alpha}
$$

Note that for $\infty>\alpha>0$, we cover the entire range of $a<x<b$. The canonical form is just $\frac{d \alpha}{\alpha}$, which can be re-written in the original co-ordinates as

$$
\frac{d \alpha}{\alpha}=\frac{d x}{x-a}-\frac{d x}{x-b}=\frac{(a-b) d x}{(x-a)(x-b)}
$$

Next, consider $0<x_{1}<x_{2}$. Once again, we can use positive variables

$$
x_{1}=\alpha_{1}, \quad x_{2}=\alpha_{1}+\alpha_{2}
$$

and the form is quite trivially

$$
\frac{d \alpha_{1}}{\alpha_{1}} \frac{d \alpha_{2}}{\alpha_{2}}=\frac{d x_{1} d x_{2}}{x_{1}\left(x_{2}-x_{1}\right)}
$$

We will henceforth skip the step of parametrization with positive variables, and also omit the measure factor in presenting results.

Next consider $0<x_{1}<x_{2}<a$, the form is

$$
\left(\frac{1}{x_{1}}-\frac{1}{x_{1}-a}\right)\left(\frac{1}{x_{2}-x_{1}}-\frac{1}{x_{2}-a}\right)=\frac{a}{x_{1}\left(x_{2}-x_{1}\right)\left(a-x_{1}\right)}
$$

This extends trivially to e.g. $0<x_{1}<x_{2}<a<x_{3}<x_{4}<b$, for which the form is

$$
\frac{a b}{x_{1}\left(x_{2}-x_{1}\right)\left(a-x_{2}\right)\left(x_{3}-a\right)\left(x_{4}-x_{3}\right)\left(b-x_{4}\right)}
$$

We will find it convenient to use a notation to represent these forms. Consider a chain of inequalities of the form $0<X_{1}<X_{2} \cdots<X_{N}$. Some of the $X$ 's are the variables our form depends on, and some are constants like $a, b$ in our previous examples. We will represent the constants by underlining the corresponding $X$ 's. In this notation, the form accompanying our two examples above are denoted as $\left[x_{1}, x_{2}, \underline{a}\right]$ and $\left[x_{1}, x_{2}, \underline{a}, x_{3}, x_{4}, \underline{b}\right]$. As yet another example,

$$
\left[x_{1}, \underline{a}, \underline{b}, x_{2}, x_{3}, \underline{c}, x_{4}\right]=\left(\frac{1}{x_{1}}-\frac{1}{x_{1}-a}\right)\left(\frac{1}{x_{2}-b}-\frac{1}{x_{2}-c}\right)\left(\frac{1}{x_{3}-x_{2}}-\frac{1}{x_{3}-c}\right)\left(\frac{1}{x_{4}-c}\right)
$$


Next, suppose we have $x_{i}, y$ with $y>x_{i}$ for all $i$. Then, if the $x^{\prime} s$ are ordered so that $x_{1}<\cdots,<x_{n}$, we have $y>x_{n}$, and the form is

$$
\left[x_{1}, \cdots, x_{n}, y\right]=\frac{1}{x_{1}} \frac{1}{x_{2}-x_{1}} \cdots \frac{1}{x_{n}-x_{n-1}} \frac{1}{y-x_{n}}
$$

Then we simply sum over all the permutations

$$
\sum_{\sigma}\left[x_{\sigma_{1}}, \cdots, x_{\sigma_{n}}, y\right]
$$

Note that individual terms in this sum have spurious poles $\left(x_{i}-x_{j}\right)$, which cancel in the sum. Indeed, in this simple case, it is trivial to do the sum explicitly, and find

$$
\frac{y^{n-1}}{\left(y-x_{1}\right)\left(y-x_{2}\right) \cdots\left(y-x_{n}\right) x_{1} \cdots x_{n}}
$$

Extremely naively, we may have expected the product in the denominator, but why is there is a factor in the numerator? The reason is that otherwise, the form would not have only logarithmic singularities! For instance, the residues on $x_{1}, \cdots, x_{n} \rightarrow 0$ would give $1 / y^{n}$; it is the numerator that makes this $1 / y$. We can extend this to $y_{I}>x_{i}$ for a collection of $m$ $y$ 's. This means that the smallest $y$ is larger than the largest $x$. Thus the form is

$$
\sum_{\sigma, p}\left[x_{\sigma_{1}}, \cdots, x_{\sigma_{n}}, y_{p_{1}} \cdots, y_{p_{m}}\right]
$$

Again the spurious poles cancel in the sum, but the forms are more interesting. In the simplest new case where $n=3, m=2$ the form is

$$
\frac{x_{1} x_{2} x_{3} y_{1}+x_{1} x_{2} x_{3} y_{2}-x_{1} x_{2} y_{1} y_{2}-x_{1} x_{3} y_{1} y_{2}-x_{2} x_{3} y_{1} y_{2}+y_{1}^{2} y_{2}^{2}}{x_{1} x_{2} x_{3}\left(y_{1}-x_{1}\right)\left(y_{1}-x_{2}\right)\left(y_{1}-x_{3}\right)\left(y_{2}-x_{1}\right)\left(y_{2}-x_{2}\right)\left(y_{3}-x_{3}\right)}
$$

Let us now consider the inequality $x, y>0$ and also $x+y<1$, or $x+y>1$. The first case is just the inside of a triangle, while the second case is a quadrilateral:

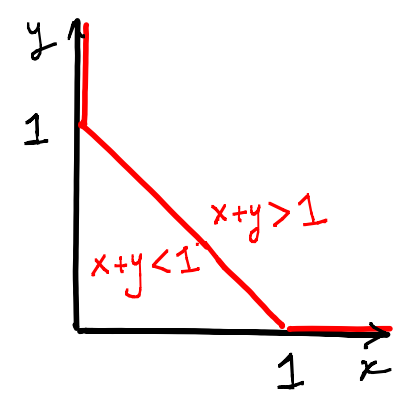

Obviously the form in the first case $x+y<1$ is

$$
\frac{-1}{x y(x+y-1)}
$$


For the second case, the region can be broken into two pieces in obvious ways. For instance, if $x>a$, there is no further restriction on $y$, while if $x<1$, we must have $y>1-x$

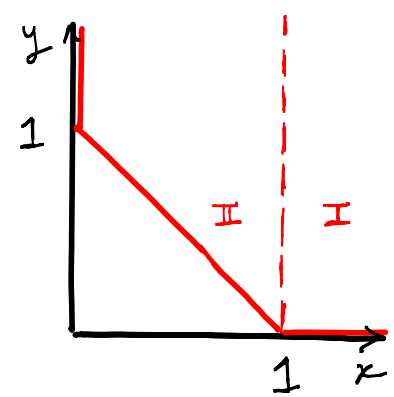

The form is then

$$
\frac{1}{x-1} \frac{1}{y}+\frac{1}{x(1-x)} \frac{1}{y+x-1}=\frac{x+y}{x y(x+y-1)}
$$

This form could have also been derived without any triangulation. The denominator reflects all the inequalities as it should. However, with a random numerator, we would have a non-vanishing residue at the origin $x=y=0$, which is clearly not in the space. The numerator kills that residue, and the resulting form has logarithmic singularities on the boundary of our space. We could have also arrived at this form in another way. We know the form for $x+y<1$. Since the form with no restriction (other than positivity) on $x, y$ is just $1 /(x y)$, we conclude that the form for $x+y>1$ is

$$
\frac{1}{x y}-\frac{-a}{x y(x+y-1)}=\frac{x+y}{x y(x+y-1)}
$$

As a final example, let us consider $x, y, a_{1}, b_{1}, a_{2}, b_{2}>0$, together with the two constraints

$$
\frac{x}{a_{1}}+\frac{y}{b_{1}}>1, \quad \frac{x}{a_{2}}+\frac{y}{b_{2}}>1
$$

We will find the form by triangulating the space in two different ways. In the first triangulation, begin by ordering $a_{1}<a_{2}$ without loss of generality; the final form will be obtained by symmetrizing $1 \leftrightarrow 2$. The shape of the allowed region in $x, y$ space depends on whether $b_{1}<b_{2}$ or $b_{1}>b_{2}$ :
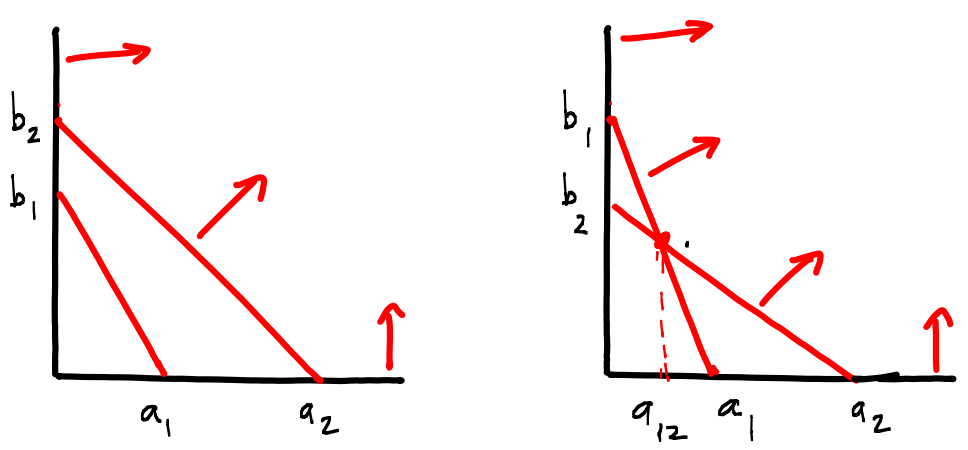
If $b_{1}<b_{2}$, then the space is essentially the same as the quadrilateral we just studied. The associated form, obtained by breaking it up into the two regions $x>a_{2}$, and $0<x<a_{2}$, is given by

$$
\left[a_{1}, a_{2}\right]\left[b_{1}, b_{2}\right]\left(\left[\underline{a_{2}}, x\right] \frac{1}{y}+\left[x, \underline{a_{2}}\right] \frac{1}{y+\frac{b_{2} x}{a_{2}}-b_{2}}\right)
$$

If $b_{1}>b_{2}$, we have a pentagonal shape. We can break this up into three regions, where $x>a_{2}, a_{2}>x>a_{12}$ and $a_{12}>x>0$. Here $a_{12}=\frac{a_{1} a_{2}\left(b_{1}-b_{2}\right)}{a_{2} b_{1}-a_{1} b_{2}}$. The associated form is

$$
\left[a_{1}, a_{2}\right]\left[b_{2}, b_{1}\right]\left(\left[\underline{a_{2}}, x\right] \frac{1}{y}+\left[\underline{a_{12}}, x, \underline{a_{2}}\right] \frac{1}{y+\frac{b_{2} x}{a_{2}}-b_{2}}+\left[x, \underline{a_{12}}\right] \frac{1}{y+\frac{b_{1} x}{a_{1}}-b_{1}}\right)
$$

Summing these forms and symmetrizing in $1 \leftrightarrow 2$, all the spurious poles cancel and we find for the final form

$$
\frac{\left(\frac{x}{a_{1}}+\frac{y}{b_{1}}\right)\left(\frac{x}{a_{2}}+\frac{y}{b_{2}}\right)}{x y a_{1} b_{1} a_{2} b_{2}\left(\frac{x}{a_{1}}+\frac{y}{b_{1}}-1\right)\left(\frac{x}{y_{2}}+\frac{y}{b_{2}}-1\right)}
$$

Note that we could also have arrived at this result in another simpler way, by thinking of the constraints in $\left(a_{1}, b_{1}\right)$ and $\left(a_{2}, b_{2}\right)$ spaces separately. For fixed $x, y$, if we redefine $A_{i}=x / a_{i}$ and $B_{i}=y / b_{i}$, we just have $A_{1}+B_{1}>1, A_{2}+B_{2}>1$. We then get for the form

$$
\frac{1}{x y} \times \frac{A_{1}+B_{1}}{A_{1} B_{1}\left(A_{1}+B_{1}-1\right)} \times \frac{\left(A_{2}+B_{2}\right)}{A_{2} B_{2}\left(A_{2}+B_{2}-1\right)}
$$

which, including the trivial Jacobian factors from the change of variables, reduces immediately to our above result obtained using triangulation.

\section{Two loops}

We now move on to studying the inequalities defining the amplituhedron for four-particle scattering, starting at two-loops, where we just have a single mutual positivity condition to deal with, simply

$$
\left(x_{1}-x_{2}\right)\left(z_{1}-z_{2}\right)+\left(y_{1}-y_{2}\right)\left(w_{1}-w_{2}\right)<0
$$

Without loss of generality we can take $x_{1}<x_{2}$. Then we have

$$
z_{1}-z_{2}>\frac{\left(y_{1}-y_{2}\right)\left(w_{1}-w_{2}\right)}{x_{2}-x_{1}}
$$

If either $y_{1}>y_{2}, w_{1}>w_{2}$ or $y_{1}<y_{2}, w_{1}<w_{2}$, we have $\left(y_{1}-y_{2}\right)\left(w_{1}-w_{2}\right)>0$; the form is then

$$
\left[x_{1}, x_{2}\right] \frac{1}{z_{2}} \frac{1}{z_{1}-z_{2}-\frac{\left(y_{1}-y_{2}\right)\left(w_{1}-w_{2}\right)}{x_{2}-x_{1}}}\left(\left[y_{1}, y_{2}\right]\left[w_{1}, w_{2}\right]+\left[y_{2}, y_{1}\right]\left[w_{2}, w_{1}\right]\right)
$$

But if $y_{1}<y_{2}, w_{1}>w_{2}$ or $y_{1}>y_{2}, w_{1}<w_{2}$, we have

$$
z_{2}-z_{1}<-\frac{\left(y_{1}-y_{2}\right)\left(w_{1}-w_{2}\right)}{x_{2}-x_{1}}
$$


Then the form is

$$
\frac{1}{x_{1}} \frac{1}{x_{2}-x_{1}} \frac{1}{z_{1}}\left(\frac{1}{z_{2}}-\frac{1}{z_{2}-z_{1}+\frac{\left(y_{1}-y_{2}\right)\left(w_{1}-w_{2}\right)}{x_{2}-x_{1}}}\right)\left(\left[y_{1}, y_{2}\right]\left[w_{2}, w_{1}\right]+\left[y_{2}, y_{1}\right]\left[w_{1}, w_{2}\right]\right)
$$

Finally, we just have to swap $1 \leftrightarrow 2$. The sum of these terms is then

$$
\frac{x_{1} z_{2}+x_{2} z_{1}+y_{1} w_{2}+y_{2} w_{1}}{x_{1} x_{2} y_{1} y_{2} z_{1} z_{2} w_{1} w_{2}\left[\left(x_{1}-x_{2}\right)\left(z_{1}-z_{2}\right)+\left(y_{1}-y_{2}\right)\left(w_{1}-w_{2}\right)\right]}
$$

We can expand it as a sum of four terms by canceling terms in numerator and denominator,

$$
\begin{aligned}
& \left(\frac{1}{x_{2} y_{1} y_{2} z_{1} w_{1} w_{2}\left[\left(x_{1}-x_{2}\right)\left(z_{1}-z_{2}\right)+\left(y_{1}-y_{2}\right)\left(w_{1}-w_{2}\right)\right]}+1 \leftrightarrow 2\right) \\
& +\left(\frac{1}{x_{1} x_{2} y_{2} z_{1} z_{2} w_{1}\left[\left(x_{1}-x_{2}\right)\left(z_{1}-z_{2}\right)+\left(y_{1}-y_{2}\right)\left(w_{1}-w_{2}\right)\right]}+1 \leftrightarrow 2\right)
\end{aligned}
$$

We can solve for all variables in terms of momentum twistors, finding

$$
\left[\begin{array}{c}
\langle 1234\rangle^{3} \\
\langle A B 12\rangle\langle A B 23\rangle\langle A B 34\rangle\langle A B C D\rangle
\end{array}+\frac{\langle 1234\rangle^{3}}{\langle C D 34\rangle\langle C D 14\rangle\langle C D 12\rangle} \quad \begin{array}{c}
\langle C D 14\rangle\langle C D 12\rangle\langle C D 23\rangle
\end{array}\right]+\text { symmetrization }
$$

where by symmetrization we mean adding another two terms where we swap $(A B) \leftrightarrow(C D)$. The expression (4.8) is the integrand for two double boxes

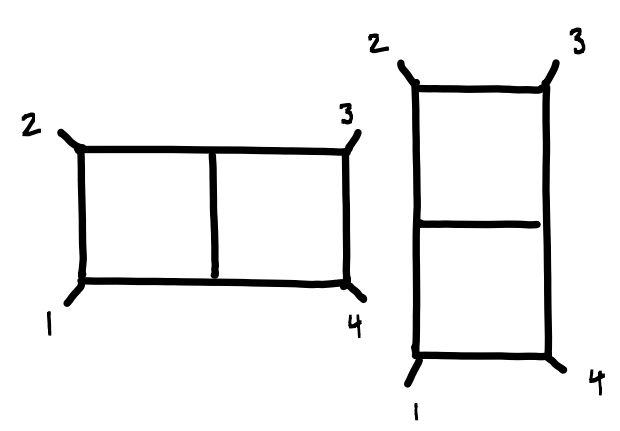

which is the standard representation of the two-loop amplitude. Note that our approach gives the fully symmetrized (in $(A B) \leftrightarrow(C D)$ ) integrand, so we get four terms instead of two.

It is natural to ask whether some other triangulation of the space may have directly given us this local expansion, but it is easy to see that this is impossible: each double box individually has a cut which is not allowed by the positivity conditions and is therefore "outside" the amplituhedron. The cut is a simple one: suppose we double cut one loop 
variable so that $D_{(1)}$ passes through the point 1 , while $D_{(2)}$ passes through the point 3. The $D$ matrices on this cut have the form

$$
D_{(1)}=\left(\begin{array}{cccc}
1 & 0 & 0 & 0 \\
0 & y & 1 & z
\end{array}\right), \quad D_{(2)}=\left(\begin{array}{cccc}
1 & x & 0 & -w \\
0 & 0 & 1 & 0
\end{array}\right)
$$

But note that the mutual positivity condition between $D_{(1)}$ and $D_{(3)}$ is automatically satisfied,

$$
\left\langle D_{(1)} D_{(2)}\right\rangle=x z+y w>0
$$

Because of this, we conclude that any further residue where $(A B)_{1}(A B)_{2}$ is cut must vanish, since there is no way to set this to zero without further setting one of $x, z$, and $y, w$, to zero.

This is a very simple and striking prediction of positivity, which is true at any loop order: if we single out any two loops $(A B)_{1},(A B)_{2}$, and consider doubly cutting each such that one line passes through 1 and the other through 3 , then the residue cutting $(A B)_{1}(A B)_{2}$ vanishes. The vanishing of this cut is not manifest from the local expansion. Even at two loops, each double box individually obviously has support on this 5 -cut, but the residue cancels in the sum

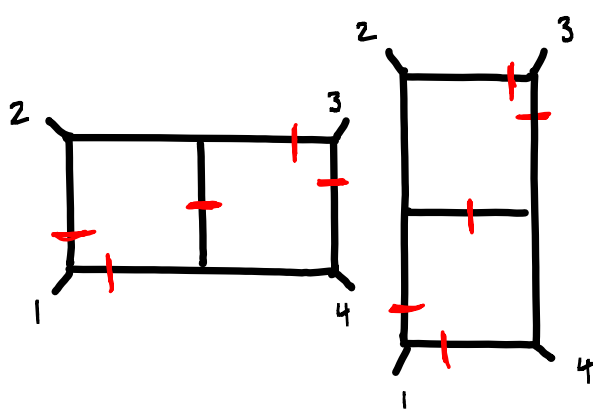

By contrast, obviously each term in our triangulation is compatible with all positivity conditions. This mirrors familiar features of the BCFW expansion for tree amplitudes: they correspond to triangulations that are "inside" the amplituhedron and manifestly consistent with positivity properties (and therefore also the symmetries of the theory), at the expense of manifest locality.

\section{$5 \quad$ Generalities on cuts}

Before starting our more detailed exploration of multiloop amplitudes, let us make some general observations about cuts of the integrand.

Reconstruction from single cuts. We are familiar with reconstructing the integrand from BCFW shifts of the external data [12]. For instance, if we shift $Z_{1} \rightarrow \hat{Z}_{1}=Z_{1}+\alpha Z_{4}$, the integrand at $\alpha=0$ is (the negative of) the residues of the single cuts where $\left\langle(A B)_{i} \hat{1} 2\right\rangle \rightarrow$ 0 . This is trivially reflected from positivity. We can divide the $w_{i}$ space into the pieces where $w_{1}$ is smallest, $w_{2}$ is smallest and so on. Suppose $w_{1}$ is smallest; then we can set $w_{i}=w_{1}+\hat{w}_{i}$. The remaining positivity conditions are then exactly the same as the 
computation of the single cut where $w_{1} \rightarrow 0$. Furthermore, we have to sum over the single cuts for setting each of the $w_{i} \rightarrow 0$.

Obviously, we can extend this to all the variables $(x, y, z, w)$. We can always take $x_{i_{x}}, y_{i_{y}}, z_{i_{z}}, w_{i_{w}}$ to be smallest, and sum over all possible $i_{x}, i_{y}, i_{z}, i_{w}$. Then, we can compute the integrand directly by summing over all these 4-cuts. This naturally corresponds to using a residue theorem using an extended BCFW deformation under which $Z_{1} \rightarrow \hat{Z}_{1}=$ $Z_{1}+\alpha Z_{4}+\beta Z_{2}, Z_{3} \rightarrow \hat{Z}_{3}=Z_{3}+\gamma Z_{2}+\rho Z_{4}$.

Emergent planarity and leading singularities. Let us now consider the opposite extreme, and look at the zero-dimensional faces of the amplituhedron. Here, each $D_{(i)}$ is taken to be one of the zero dimensional cells of $G(2,4)$, where the columns $i, j$ can be set to the identity and the remaining entries are zero. From the mutual positivity of equation (4.1), we learn something very simple right away: the configuration will satisfy positivity in all cases except one: we can't have the (13) cells and (24) cells at the same time. It is trivial to see that this fact extends to all $n$ MHV amplitudes at all loop orders. If all the $A B_{i}=(a b)_{i}$ are drawn as chords on a disk, then a configuration with lines that don't cross is allowed, but a configuration with lines that cross violates positivity and must have vanishing residue. Examples of allowed and non-allowed configurations are shown below:

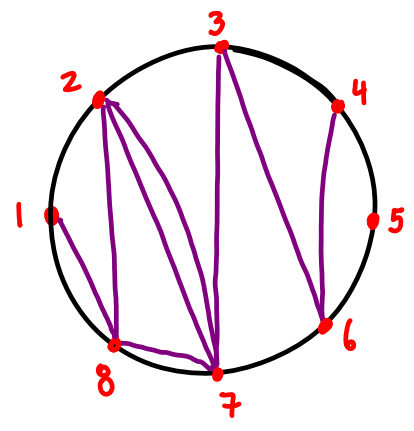

ALLOWED

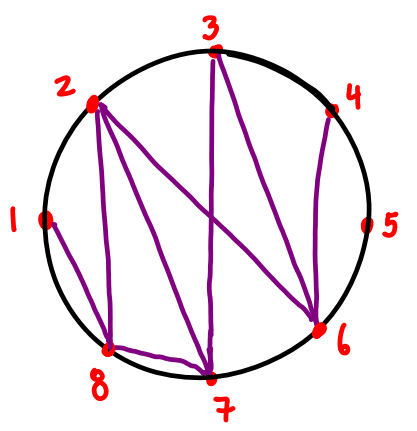

NOT ALLOWED

The fact that our form can ultimately be expressed as a sum over planar local integrands is not obviously built into the geometry, but of course does emerge from it. We see this planarity very vividly in the above structure of leading singularities - clearly planar diagrams can only give us leading singularities of the allowed type, while all other objects can give us the illegal "crossing" configurations. Indeed there are many meaningful local integrands, compatible with the cyclic structure on external data, which can nonetheless not be considered as "planar". A simple example is the square of one-loop amplitude, whose integrand can be written in momentum twistor space as

$$
\frac{\langle 1234\rangle^{4}}{\langle A B 12\rangle\langle A B 23\rangle\langle A B 34\rangle\langle A B 14\rangle\langle C D 12\rangle\langle C D 23\rangle\langle C D 34\rangle\langle C D 14\rangle}
$$

This integrand has an obvious leading singularity where e.g. $A B=13$ and $C D=24$, which cross in index space. This is a "not allowed" leading singularity that is incompatible with 
positivity. Thus, we see that the planar structure of the integrand is not a trivial consequence of cyclically ordered external data, but actually emerges from the positive geometry of the amplituhedron. Note that "planarity" is not an obvious invariant property of the full integrand, but is only a natural statement about a particular expansion of the integrand in terms of (local) Feynman diagrams. It is thus perhaps not surprising that planarity should be one of many derived properties of the integrand from the amplituhedron point of view.

\section{Unitarity from positivity}

In much of the recent work on scattering amplitudes in planar $\mathcal{N}=4 \mathrm{SYM}$, the unitarity of loop amplitudes has been directly associated with correctly matching the single cut of the loop integrand [12], determined by the the forward limit [13] of the lower-loop amplitude. However there is an even simpler manifestation of unitarity, familiar from the textbooks, in the double-cut (or "unitarity cut") of the integrand, which is given by sewing together two lower loop integrand.
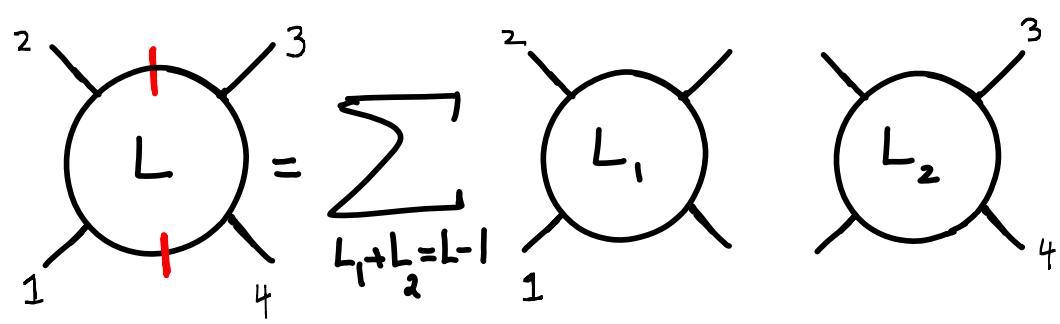

This is easy to translate to momentum-twistor language. Starting with the $L$-loop integrand, we take one loop variable, $(A B)_{L}$, to cut (12) and (34). The corresponding $D$ matrix is then of the form

$$
D_{L}=\left(\begin{array}{llll}
1 & x & 0 & 0 \\
0 & 0 & y & 1
\end{array}\right)
$$

If we compute the residue of the integrand on this configuration, unitarity tells us that the result must be

$$
\frac{d x}{x} \frac{d y}{y} \times \sum_{L_{1}+L_{2}=L-1} M_{4}^{L_{1}}\left(Z_{1}-x Z_{2}, Z_{2}, Z_{3}, Z_{4}-y Z_{3}\right) M_{4}^{L_{2}}\left(Z_{1}, Z_{2}-\frac{1}{x} Z_{1}, Z_{3}-\frac{1}{y} Z_{4}, Z_{4}\right)
$$

We will now see that this result follows in a simple and beautiful way from the positive geometry of the amplituhedron.

On the unitarity cut, the positivity conditions are the usual ones for the $(L-1)$ loop variables. For $D_{L}$ we just have that $x, y>0$. The mutual positivity between $D_{L}$ and the remaining $D_{i}$ just tells us that

$$
(23)_{i}+x y(14)_{i}-x(13)_{i}-y(24)_{i}>0
$$

This condition also tells us that

$$
\begin{aligned}
{[(13)-y(14)][(24)} & -x(14)]=(13)(24)-x(13)(14)-y(14)(24)+x y(14)^{2} \\
& =(12)(34)+(14)[(23)+x y(14)-x(13)-y(24)]>(12)(34)>0
\end{aligned}
$$


where in the second line we used $(13)(24)=(12)(34)+(23)(14)$. Now, obviously we can divide the space of each $D_{i}$ into ones where $(13)_{i}-(14)_{i}>0$, and $(13)_{i}-y(14)_{i}<0$, similarly $(24)_{i}-x(14)_{i}>0$ or $(24)_{i}-x(14)_{i}<0$. However, if the product of these two factors is negative it is impossible to satisfy equation (6.3). Thus, for each $i$, we have either that

$$
(13)-y(14)>0 \quad \text { and } \quad(24)-x(14)>0
$$

or

$$
(13)-y(14)>0, \quad \text { and } \quad(24)-x(14)>0
$$

Let us say that $L_{1}$ of the lines $D_{a}$ satisfy the first inequality and the remaining $L_{2}=$ $L-1-L_{1}$ lines $D_{A}$ satisfy the second inequality. Explicitly, in the first case the region is represented by positivity conditions

$$
\begin{gathered}
(12)_{a}>0,(13)_{a}-y(14)_{a}>0,(14)_{a}>0,(23)_{a}>0,(24)_{a}-x(14)_{a}>0,(34)_{a}>0 \\
(23)_{a}+x y(14)_{a}-x(13)_{a}-y(24)_{a}>0
\end{gathered}
$$

Let us define shifted columns

$$
(\hat{3})_{a}=(3)_{a}-y(4)_{a}, \quad(\hat{2})_{a}=(2)_{a}-x(1)_{a}
$$

Thus the set of positivity conditions become

$$
(1 \hat{2})_{a}>0,(1 \hat{3})_{a}>0,(14)_{a}>0,(\hat{2} \hat{3})_{a}>0,(\hat{2} 4)_{a}>0,(\hat{3} 4)_{a}>0
$$

In the second region we have

$$
\begin{gathered}
(12)_{A}>0, y(14)_{A}-(13)_{A}>0,(14)_{A}>0,(23)_{A}>0, x(14)_{A}-(24)_{A}>0,(34)_{A}>0 \\
(23)_{A}+x y(14)_{A}-x(13)_{A}-y(24)_{A}>0
\end{gathered}
$$

Let us define shifts

$$
(\hat{1})_{A}=(1)_{A}-\frac{1}{x}(2)_{A}, \quad(\hat{4})_{A}=(4)_{A}-\frac{1}{y}(3)_{A}
$$

Then the set of positivity conditions become

$$
(\hat{1} 2)_{A}>0,(\hat{1} 3)_{A}>0,(\hat{1} \hat{4})_{A}>0,(23)_{A}>0,(2 \hat{4})_{A}>0,(3 \hat{4})_{A}>0
$$

Now, we come to the positivity conditions internal to the $D_{a}$ 's, internal to the $D_{A}$ 's, and also the ones between $D_{a}$ and $D_{A}$ 's. Actually quite strikingly, the $D_{a}$ 's and $D_{A}$ 's are automatically mutually positive! We look at

$$
(12)_{a}(34)_{A}+(23)_{a}(14)_{A}+(34)_{a}(12)_{A}+(14)_{a}(23)_{A}-(13)_{a}(24)_{A}-(24)_{a}(13)_{A}
$$

Rewriting this in terms of the natural shifted variables

$$
(2)_{a}=(\hat{2})_{a}+x(1)_{a}, \quad(3)_{a}=(\hat{3})_{a}+y(4)_{a} \quad(1)_{A}=(\hat{1})_{A}+\frac{1}{x}(2)_{A}, \quad(4)_{A}=(\hat{4})_{A}+\frac{1}{y}(3)_{A}
$$


and plugging into (6.13) we find

$$
\begin{aligned}
& (1 \hat{2})_{a}(3 \hat{4})_{A}+\left[(\hat{2} \hat{3})_{a}+x y(14)_{a}+y(\hat{2} 4)_{a}+x(1 \hat{3})_{a}\right]\left[\frac{1}{x y}(23)_{A}+(\hat{1} \hat{4})_{A}+\frac{1}{x}(2 \hat{4})_{A}+\frac{1}{y}(\hat{1} 3)_{A}\right] \\
& +(\hat{3} 4)_{a}(\hat{1} 2)_{A}+(14)_{a}(23)_{A}-\left[(1 \hat{3})_{a}+y(14)_{a}\right]\left[(2 \hat{4})_{A}+\frac{1}{y}(23)_{A}\right]-\left[(\hat{2} 4)_{a}+x(14)_{a}\right]\left[(\hat{1} 3)_{A}+\frac{1}{x}(23)_{A}\right] \\
& =(1 \hat{2})_{a}(3 \hat{4})_{A}+(\hat{3} 4)_{a}(\hat{1} 2)_{A}+\frac{1}{y}\left[(\hat{2} \hat{3})_{a}+x(1 \hat{3})_{a}\right](\hat{1} 3)_{A}+x\left[(1 \hat{3})_{a}+y(14)_{a}\right](\hat{1} \hat{4})_{A} \\
& \quad+\left[(\hat{2} \hat{3})_{a}+y(\hat{2} 4)_{i}\right](14)_{A}+\frac{1}{x}\left[(\hat{2} \hat{3})_{a}+y(\hat{2} 4)_{i}\right](2 \hat{4})_{A}+\frac{1}{x y}(\hat{2} \hat{3})_{a}(23)_{A}>0
\end{aligned}
$$

The positivity here is quite non-trivial; the expression many terms with plus and minus signs that cancel each other, leaving only pluses.

The mutual positivity internally for the $D_{a}$ 's (or the $D_{A}$ 's) are exactly the same for the shifted and unshifted columns, since the $(4 \times 4)$ determinants are unchanged in shifting a column by a multiple of another. These can be easily translated in shifts of external twistors. Under $\mathcal{A}_{\gamma}=D_{\gamma a} \cdot Z_{a}$, we have for the first shift

$$
\begin{aligned}
A=D \cdot Z & =(1) Z_{1}+(\hat{2}) Z_{2}+(\hat{3}) Z_{3}+(4) Z_{4} \\
& =(1) Z_{1}+(2) Z_{2}-x(1) Z_{2}+(3) Z_{3}-y(4) Z_{3}+(4) Z_{4} \\
& =(1) \hat{Z}_{1}+(2) Z_{2}+(3) Z_{3}+(4) \hat{Z}_{4}
\end{aligned}
$$

Thus, the form for the $L_{1}$ lines is

$$
M_{4}^{L_{1}}\left(Z_{1}-x Z_{2}, Z_{2}, Z_{3}, Z_{4}-y Z_{3}\right)
$$

and analogously the form for the $L_{2}$ lines is

$$
M_{4}^{L_{2}}\left(Z_{1}, Z_{2}-\frac{1}{x} Z_{1}, Z_{3}-\frac{1}{y} Z_{4}, Z_{4}\right)
$$

Thus, we conclude that the unitarity cut is

$$
\frac{d x}{x} \frac{d y}{y} \times \sum_{L_{1}+L_{2}=L-1} M_{4}^{L_{1}}\left(Z_{1}-x Z_{2}, Z_{2}, Z_{3}, Z_{4}-y Z_{3}\right) M_{4}^{L_{2}}\left(Z_{1}, Z_{2}-\frac{1}{x} Z_{1}, Z_{3}-\frac{1}{y} Z_{4}, Z_{4}\right)
$$

precisely as needed to enforce unitarity.

\section{Three loops}

Having established these general results, let us turn to the three-loop amplitude. Recall from our general discussion that it suffices to look at various cuts of the amplitude, coming from taking $x_{\sigma_{1}}, y_{\sigma_{2}}, z_{\sigma_{3}}, w_{\sigma_{4}}$ to be smallest. For the case of three loops, at least one pair of $\sigma_{1}, \sigma_{2}, \sigma_{3}, \sigma_{4}$ will correspond to the same loop, thus, to compute the full three-loop integrand, it suffices to compute the cut of the integrand where one loop is double-cut. We have already verified that the unitarity double-cut is correctly reproduced at any loop order. It thus suffices to compute the remaining double cuts, which we call the "corner cuts": where the line passes through one of the points $Z_{i}$, or its parity conjugate, where the 
line lies in the plane $\left(Z_{i-1} Z_{i} Z_{i+1}\right)$. Since these are parity conjugate, it is enough to compute one of them, which we take to be the cut where the line corresponding to the third loop passes through point 4 . It will be convenient to use a different gauge-fixing for the third loop

$$
D_{(3)}=\left(\begin{array}{llll}
a & 1 & b & 0 \\
0 & 0 & 0 & 1
\end{array}\right)
$$

If we further rescale the variables for the remaining loop variables as $w_{i} \rightarrow w_{i} / b, y_{i} \rightarrow$ $b y_{i} ; z_{i} \rightarrow z_{i} / a, x_{i} \rightarrow a x_{i}$, the remaining positivity conditions become

$$
x_{i}+y_{i}>1, \quad\left(x_{1}-x_{2}\right)\left(z_{1}-z_{2}\right)+\left(y_{1}-y_{2}\right)\left(w_{1}-w_{2}\right)<0
$$

We can assume that $x_{1}<x_{2}$, so that just as for two-loops we have then sum at the end over $1 \leftrightarrow 2$. Let us also define

$$
Z_{+}=\frac{1}{z_{2}} \frac{1}{z_{1}-z_{2}-\frac{\left(y_{1}-y_{2}\right)\left(w_{1}-w_{2}\right)}{x_{2}-x_{1}}}, \quad Z_{-}=\frac{1}{z_{1}}\left(\frac{1}{z_{2}}-\frac{1}{z_{2}-z_{1}-\frac{\left(y_{2}-y_{1}\right)\left(w_{1}-w_{2}\right)}{x_{2}-x_{1}}}\right)
$$

Then, by dividing the space into pieces much as we did at 2-loops, we find that the form is

$$
\begin{aligned}
& {\left[x_{1}, x_{2}, \underline{1}\right]\left(\left[\underline{1-x_{1}}, y_{1}, y_{2}\right]\left(\left[w_{1}, w_{2}\right] Z_{+}+\left[w_{2}, w_{1}\right] Z_{-}\right)\right.} \\
& \left.+\left(\left[\underline{1-x_{2}}, y_{2}, \underline{1-x_{1}}, y_{1}\right]+\left[\underline{1-x_{1}}, y_{2}, y_{1}\right]\right)\left(\left[w_{2}, w_{1}\right] Z_{+}+\left[w_{1}, w_{2}\right] Z_{-}\right)\right) \\
& +\left[x_{1}, \underline{1}, x_{2}\right]\left(\left[\underline{1-x_{1}}, y_{1}, y_{2}\right]\left(\left[w_{1}, w_{2}\right] Z_{+}+\left[w_{2}, w_{1}\right] Z_{-}\right)\right. \\
& \left.+\left(\left[y_{2}, \underline{1-x_{1}}, y_{1}\right]+\left[\underline{1-x_{1}}, y_{2}, y_{1}\right]\right)\left(\left[w_{2}, w_{1}\right] Z_{+}+\left[w_{1}, w_{2}\right] Z_{-}\right)\right) \\
& +\left[\underline{1}, x_{1}, x_{2}\right]\left(\left[y_{1}, y_{2}\right]\left(\left[w_{1}, w_{2}\right] Z_{+}+\left[w_{2}, w_{1}\right] Z_{-}\right)+\left[y_{2}, y_{1}\right]\left(\left[w_{2}, w_{1}\right] Z_{+}+\left[w_{1}, w_{2}\right] Z_{-}\right)\right.
\end{aligned}
$$

Adding $1 \leftrightarrow 2$, all spurious poles cancel and we obtain

$$
\frac{\left\{\begin{array}{c}
w_{2} x_{1} x_{2} y_{1}+w_{2} x_{2} y_{1}^{2}+w_{1} x_{1} x_{2} y_{2}-w_{1} y_{1} y_{2}-w_{2} y_{1} y_{2}+w_{2} x_{1} y_{1} y_{2}+w_{1} x_{2} y_{1} y_{2} \\
+w_{2} y_{1}^{2} y_{2}+w_{1} x_{1} y_{2}^{2}+w_{1} y_{1} y_{2}^{2}-x_{1} x_{2} z_{1}+x_{1} x_{2}^{2} z_{1}+x_{2}^{2} y_{1} z_{1}+x_{1} x_{2} y_{2} z_{1} \\
+x_{2} y_{1} y_{2} z_{1}-x_{1} x_{2} z_{2}+x_{1}^{2} x_{2} z_{2}+x_{1} x_{2} y_{1} z_{2}+x_{1}^{2} y_{2} z_{2}+x_{1} y_{1} y_{2} z_{2}
\end{array}\right\}}{a b x_{1} x_{2} y_{1} y_{2} z_{1} z_{2} w_{1} w_{2}\left(x_{1}+y_{1}-1\right)\left(x_{2}+y_{2}-1\right)\left(\left(x_{2}-x_{1}\right)\left(z_{1}-z_{2}\right)+\left(y_{2}-y_{1}\right)\left(w_{1}-w_{2}\right)\right)}
$$


This matches what we get from the familiar local expansion, as a sum over ladders and "tennis court" diagrams:
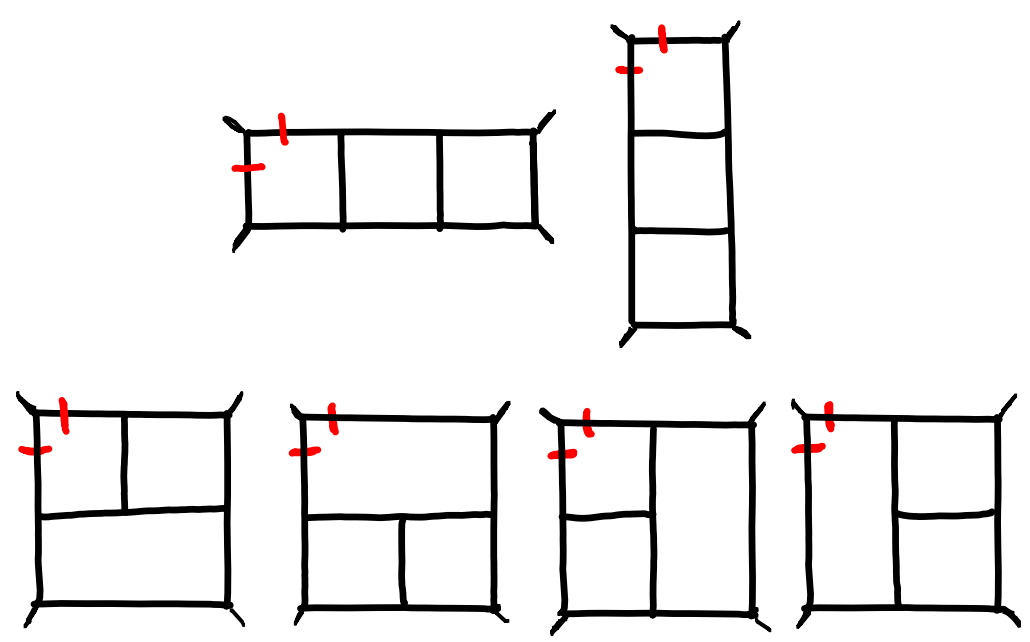

\section{Multi-collinear region}

We have seen that a particular double cut of a single loop - the unitarity cut — is simply expressed in terms of (shifted) lower-loop objects. It is thus natural to look at the other two kinds of double cuts. Let us consider the cut where the $L^{t h}$ line passes through 2 . It will be convenient to use a different gauge-fixing for this last line

$$
D_{(L)}=\left(\begin{array}{cccc}
0 & 1 & 0 & 0 \\
-\alpha & 0 & 1 & \gamma
\end{array}\right)
$$

Then the mutual positivity conditions between $D_{(L)}$ and the other lines is simply

$$
\alpha w_{i}+z_{i}>\gamma
$$

It is amusing that from the point of view of the lower-loop problem, we are simply putting a simple additional restriction on the allowed region for $w_{i}, z_{i}$.

Despite the apparent simplicity of this deformation of the $L-1$ loop problem, unlike the unitarity cut, this double cut can't be determined in terms of shifts of lower-loop problems in a straightforward way. However, there is a further, triple cut, which does have a very simple interpretation. Consider the limit where $\beta \rightarrow 0$. This is the collinear region, where the line passes through the point 2 while lying in the plane (123) [14]. Note that the positivity condition is now automatically satisfied, and so the cut is trivial:

$$
A_{L}^{\text {coll. }}=\frac{d \alpha}{\alpha} \times A_{L-1}
$$

In this discussion we assumed that all the lines but one are generic. We now investigate what happens when $l$ lines are sent into the collinear region. The most general way this can 
happen is to start with $L_{12}$ lines cutting (12) and $L_{23}$ lines cutting (23). Let us gauge-fix in a convenient way, and write for the two sets of lines

$$
D_{(i)}=\left(\begin{array}{cccc}
\beta_{i} & 1 & 0 & 0 \\
-\alpha_{i} & 0 & 1 & \gamma_{i}
\end{array}\right) \quad D_{(I)}=\left(\begin{array}{cccc}
0 & 1 & \rho_{I} & 0 \\
\alpha_{I} & 0 & 1 & \gamma_{I}
\end{array}\right)
$$

In order to reach the collinear limit, we must send $\beta_{i}, \gamma_{i} \rightarrow 0$, and $\rho_{I}, \delta_{I} \rightarrow 0$. We can send these to zero in different ways, but let us focus on one for definiteness, the other cases can be treated similarly. For the lines cutting (12), we first take them to pass through 2, and then move them into the collinear region where they lie in (123); in other words, we first send $\beta_{i} \rightarrow 0$, and then $\gamma_{i} \rightarrow 0$. Similarly for the lines intersecting (23), we first send them to pass through 2 , then to lie in (123), so that we put the $\rho_{I} \rightarrow 0$, then send $\gamma_{I} \rightarrow 0$. Now, the positivity conditions between these lines are just

$$
\left(\beta_{i}-\beta_{j}\right)\left(\gamma_{i}-\gamma_{j}\right)>0, \quad\left(\rho_{I}-\rho_{J}\right)\left(\frac{\gamma_{I}}{\alpha_{I}}-\frac{\gamma_{J}}{\alpha_{J}}\right)>0, \quad\left(\beta_{i}-\alpha_{I} \rho_{I}\right)\left(\gamma_{i}-\gamma_{I}\right)>0
$$

Collectively, these tell us something simple. Suppose we take the lines to pass through 2 in some particular order, say by first taking $\beta_{1} \rightarrow 0$, then $\beta_{2} \rightarrow 0$, then $\rho_{1} \rightarrow 0$, then $\rho_{2} \rightarrow 0$, then $\beta_{3} \rightarrow 0$ etc. Then, the cut vanishes unless the lines are taken into the collinear limit in exactly the same order! In this case, the cut is just

$$
\prod_{a=1}^{l} \frac{d \alpha_{a}}{\alpha_{a}} \times M^{L-l}
$$

\section{$9 \quad$ Log of the amplitude}

Scattering amplitudes have well-known double-logarithmic infrared divergences, arising precisely from loop integration in the collinear region. At $L$ loops, we have a $\log ^{2 L}$ divergence, which exponentiates in a well-known way; the logarithm of the amplitude only has a $\log ^{2}$ divergence. This is a motivation for looking at the $\log$ of the amplitude from a physical point of view. But as we have just seen, the loop integrand form also has an extremely simple behavior in the multicollinear limit. We will now see that this behavior, together with some very simple combinatorics, already motivates looking at the logarithm of the amplitude directly at the level of the integrand. While the amplitude itself has a non-vanishing residue when one loop momentum is brought into the collinear region, we will see that the $\log$ of the amplitude vanishes in the multicollinear region, unless all $L$ loop momenta are taken into the collinear region. The residue depends in a non-trivial way on the specific path taken into the collinear region. Furthermore, we will see that the log of the amplitude naturally leads us to consider all the natural "positive regions" we can think of related to amplituhedron geometry.

Let us start by introducing a generating function combining together the amplitude at all loop order, otherwise known as the amplitude itself:

$$
M=1+g M_{1}+g^{2} M_{2}+\cdots
$$


Now, consider for any function $f$, the expansion for $f(M)$. Suppose that

$$
f(1+x)=x+a_{2} x^{2}+a_{3} x^{3}+\cdots
$$

then

$$
\begin{aligned}
f(M) & =\left(g M_{1}+g^{2} M_{2}+\cdots\right)+a_{2}\left(g^{2} A_{1}^{2}+2 g^{3} M_{1} M_{2}+\cdots\right)+a_{3} g^{3} M_{3}+\cdots \\
& =g M_{1}+g^{2}\left(M_{2}+a_{2} M_{1}^{2}\right)+g^{3}\left(M_{3}+2 a_{2} M_{1} M_{2}+a_{3} M_{1}^{3}\right)+\cdots
\end{aligned}
$$

We'd now like to extract the permutation-invariant integrand from this expression at $L$ loops. For instance,

$$
\begin{aligned}
M_{3} & =\int d^{4} x_{1} d^{4} x_{2} d^{4} x_{3} M_{3}\left(x_{1}, x_{2}, x_{3}\right) \\
M_{1} M_{2} & =\int d^{4} x_{1} d^{4} x_{2} d^{4} x_{3}\left[\left(M_{1}\left(x_{1}\right) M_{2}\left(x_{2}, x_{3}\right)+M_{1}\left(x_{2}\right) M_{2}\left(x_{1}, x_{3}\right)+M_{1}\left(x_{3}\right) M_{2}\left(x_{1}, x_{2}\right)\right]\right. \\
M_{1}^{3} & =\int d^{4} x_{1} d^{4} x_{2} d^{4} x_{3} M_{1}\left(x_{1}\right) M_{1}\left(x_{2}\right) M_{1}\left(x_{3}\right)
\end{aligned}
$$

Actually, for the combinatorics, the " $\int d^{4} x$ " are irrelevant. Instead, we define a generating function

$$
M=1+\sum_{i} x_{i}(i)+\sum_{i<j} x_{i} x_{j}(i j)+\sum_{i<j<k} x_{i} x_{j} x_{k}(i j k)+\cdots
$$

Here "( $(1)$ " stands for $M_{1}\left(x_{1}\right)$, "(134)" stands for $M_{3}\left(x_{1}, x_{3}, x_{4}\right)$ and so on. Then, the integrand for $f(M)$ at $L$ loops is just the coefficient of $\left(x_{1} \cdots x_{L}\right)$ in the expansion of $f(A)$, or put another way

$$
f(M)^{L-l o o p}=\left.\partial_{x_{1} \cdots x_{L}} f(M)\right|_{x_{1}=\cdots=x_{L}=0}
$$

Obviously, if we are only interested in $L$ loops, we can truncate the $x_{i}$ to just $x_{1}, \cdots, x_{L}$ if we like. Thus, explicitly, for $L=3$, we have

$$
M=1+x_{1}(1)+x_{2}(2)+x_{3}(3)+x_{1} x_{2}(12)+x_{1} x_{3}(13)+x_{2} x_{3}(23)+x_{1} x_{2} x_{3}(123)
$$

and foreseeing our future interest, for $f(M)=\log (M)$, we have for the 3-loop log of the amplitude

$$
(\log M)^{3-l o o p}=(123)+2(1)(2)(3)-[(1)(23)+(2)(13)+(3)(12)]
$$

We would now like to compute the cut of $f(M)$ in the multi-collinear limit. Suppose $L$ lines are sent to pass through 2 in some order $(1, \cdots, L)$. We already know that the cut of the amplitude in the multi-collinear limit depends on the order in which the lines are then sent to the collinear region - indeed for the amplitude the cut vanishes unless the lines are sent to the collinear limit in the same order $(1, \cdots, L)$. This will not in general be true for $f(M)$. Suppose that a set of $l$ lines are moved into the collinear limit in some order $\sigma=\left\{\sigma_{1}, \cdots, \sigma_{l}\right\}$. For instance for $L=3$, we could take $\sigma=\{2\}$ or $\sigma=\{13\}$ or $\sigma=\{231\}$. Then, it is easy to see that the multi-collinear cut of $f(M)$ can be computed as follows. 
We first play the following game, to produce a new generating function $M^{\sigma}$ : (I) if an ordered subset of $\sigma$ occurs out of order in the brackets of $M$, we drop that term. (II) We then delete all the labels in $\sigma$. Let's illustrate this for $L=3$ with $\sigma=\{31\}$. The terms $x_{1} x_{3}(13)$ and $x_{1} x_{2} x_{3}(123)$ in $M$ are dropped, and the rest are kept. Then, we drop the indices 3,1 , and are left with

$$
M^{\{31\}}=1+x_{1}+x_{2}(2)+x_{3}+x_{1} x_{2}(2)+x_{2} x_{3}(2)
$$

The multi-collinear cut of $f(M)$ is easily seen to be just

$$
\prod \frac{d \alpha_{\sigma_{i}}}{\alpha_{\sigma_{i}}} \times\left.\partial_{x_{1}, \cdots x_{L}} f\left(M^{\sigma}\right)\right|_{x_{1}=\cdots=x_{L}=0}
$$

We are now ready to see why the logarithm of the amplitude is so natural from a purely combinatorial point of view. Let us return to looking at $M^{\{31\}}$. Observe that while $M$ is itself an irreducible polynomial, $M^{\{31\}}$ factorizes as

$$
M^{\{31\}}=\left[1+x_{1}+x_{3}\right]\left[1+x_{2}(2)\right]
$$

Note that the second factor is just the $M$ polynomial made of the undeleted variables, while the first factor is the generating function made of the variables with only terms in correct order kept.

This is a general statement. For any $\sigma$, let $\bar{\sigma}$ be the complementary set. Then

$$
M^{\sigma}=\left(1+\sum_{i} x_{\sigma_{i}}+\sum_{i<j ; \sigma_{i}<\sigma_{j}} x_{\sigma_{i}} x_{\sigma_{j}}+\cdots\right) \times M^{\bar{\sigma}}
$$

To illustrate with a more non-trivial example, say for $L=5$ and $\sigma=\{415\}$, we have

$$
M^{\{415\}}=\left(1+x_{1}+x_{4}+x_{5}+x_{1} x_{5}+x_{4} x_{5}\right) \times\left(1+x_{2}(2)+x_{3}(3)+x_{2} x_{3}(23)\right)
$$

Note that if $\sigma$ is anything other than the empty set, the factorization in non-trivial. Because of this factorization, it is natural to consider the log of the object. Then, we see that for any string $\sigma$ of length $0<l<L$,

$$
\partial_{x_{1} \cdots x_{L}} \log M^{\sigma}=0
$$

We thus learned something remarkable: if we take the log of the amplitude, then the cut taking any number $l<L$ of the loop variables into the collinear region vanishes! Only if all $L$ lines are taken into the collinear region together, can we get something non-zero. This explains why the $\log$ of the amplitude only has a $\log ^{2}$ divergence. We get a $\log ^{2}$ divergence from each loop momentum brought into this collinear region. For the amplitude itself, we can bring all $L$ lines into the collinear region one at a time, and thus we get the $\log ^{2 L}$ IR divergence. However for the log of the amplitude, since all $L$ lines must be brought in the collinear region together we just get a single overall $\log ^{2}$ divergence. (Note that had this limit given us no residue, the log would have have been completely IR divergence free!). 
Very interestingly, the logarithm of the amplitude doesn't have the property, familiar for the amplitude itself, of having "unit leading singularities". If all $L$ lines are taken into the collinear region in an order $\sigma=\left(\sigma_{1}, \cdots, \sigma_{L}\right)$, then the residue is

$$
\left.\partial_{x_{1} \cdots x_{L}} \log \left(1+\sum_{i} x_{i}+\sum_{i<j ; \sigma_{i}<\sigma_{j}} x_{i} x_{j}+\cdots\right)\right|_{x_{i}=0}
$$

Note that unlike the amplitude itself, which is only non-vanishing for $\sigma=(1,2, \cdots, n)$, the $\log$ of the amplitude vanishes in this case, since

$$
\left(1+\sum_{i} x_{i}+\sum_{i<j ; \sigma_{i}<\sigma_{j}} x_{i} x_{j}+\cdots\right)=\left(1+x_{1}\right) \cdots\left(1+x_{n}\right)
$$

maximally factorizes! In the other extreme, if $\sigma=(n, \cdots, 1)$ is oppositely ordered to $(1, \cdots, n)$, then we have

$$
\left.\partial_{x_{1} \cdots x_{L}}\left(1+x_{1}+\cdots x_{L}\right)\right|_{x_{i}=0}=(L-1) !
$$

In general, we can find residues ranging from 1 to $(L-1)$ !. For instance, at 4 loops, we have the non-vanishing residues $1,2,3,4$ and 6 , coming from the following paths:

$$
\begin{aligned}
& 1:(2,3,4,1)(2,4,1,3)(3,1,4,2)(4,1,2,3) \quad 2:(2,4,3,1)(3,2,4,1)(4,1,3,2)(4,2,1,3) \\
& 3:(3,4,1,2) \quad 4:(3,4,2,1)(4,2,3,1)(4,3,1,2) \quad 6:(4,3,2,1) \quad 0: \text { other }
\end{aligned}
$$

The log of the amplitude has another fascinating feature, which we can see already starting at 2-loops, where

$$
\log M_{2}=(12)-(1)(2)
$$

Note that the 2-loop amplitude puts the positivity restriction $\left\langle D_{(1)} D_{(2)}\right\rangle>0$ on the lines, but the one-loop-squared part does not put any positivity restrictions on them. Indeed, we can think of this as the sum over two regions, with $\left\langle D_{(1)} D_{(2)}\right\rangle>0$ and $\left\langle D_{(1)} D_{(2)}\right\rangle<0$. Thus, the sum that gives the $\log$ is the form associated with the region where $\left\langle D_{(1)} D_{(2)}\right\rangle<0$ ! The pattern continues at all higher loops. At 3-loops we have three positivity conditions involving

$$
\left\{\left\langle D_{(1)} D_{(2)}\right\rangle,\left\langle D_{(1)} D_{(3)}\right\rangle,\left\langle D_{(2)} D_{(3)}\right\rangle\right\}
$$

For the amplitude they are all positive, $M_{3}=\{+++\}$ while for the log of the amplitude (9.8) we get a sum of terms

$$
(\log M)^{3-l o o p}=\{+--\} \oplus\{-+-\} \oplus\{--+\} \oplus 2\{---\}
$$

At 4 loops we have 6 positivity conditions,

$$
\left\{\left\langle D_{(1)} D_{(2)}\right\rangle,\left\langle D_{(1)} D_{(3)}\right\rangle,\left\langle D_{(1)} D_{(4)}\right\rangle,\left\langle D_{(2)} D_{(3)}\right\rangle,\left\langle D_{(2)} D_{(4)}\right\rangle,\left\langle D_{(3)} D_{(4)}\right\rangle\right\}
$$


For the amplitude we have $M_{4}=\{++++++\}$. The log is

$$
\begin{aligned}
(\log M)^{4-l o o p}=(1234)-[(12)(34)+(13)(24)+(14)(23)] \\
\quad+[(12)(3)(4)+(13)(2)(4)+(14)(2)(3)+(23)(1)(4)+(24)(1)(3)+(34)(1)(2)] \\
\quad+2[(123)(4)+(124)(3)+(134)(2)+(234)(1)]-6(1)(2)(3)(4)
\end{aligned}
$$

and can be decomposed into a sum of regions as

$$
(\log M)^{4-\text { loop }}=R_{1} \oplus 2 R_{2} \oplus 3 R_{3} \oplus 4 R_{4} \oplus 6 R_{6}
$$

where

$$
\begin{aligned}
R_{1}= & \{---+++\} \oplus\{--++-+\} \oplus\{--+++-\} \oplus\{-+--++\} \\
& \oplus\{-+-++-\} \oplus\{-++--+\} \oplus\{-++-+-\} \oplus\{-+++--\} \\
& \oplus\{+---++\} \oplus\{+--+-+\} \oplus\{+-+--+\} \oplus\{+-+-+-\} \\
& \oplus\{+-++--\} \oplus\{++---+\} \oplus\{++--+-\} \oplus\{++-+--\} \\
R_{2}= & \{----++\} \oplus\{---+-+\} \oplus\{---++-\} \oplus\{--+--+\} \\
& \oplus\{--+-+-\} \oplus\{-+---+\} \oplus\{-+-+--\} \oplus\{-++---\} \\
& \oplus\{+---+-\} \oplus\{+--+--\} \oplus\{+-+---\} \oplus\{++----\} \\
R_{3}= & \{--++--\} \oplus\{-+--+-\} \oplus\{+----+\} \\
R_{4}= & \{-----+\} \oplus\{----+-\} \oplus\{---+--\} \oplus\{--+---\} \\
& \oplus\{-+----\} \oplus\{+-----\} \\
R_{6}= & \{------\}
\end{aligned}
$$

While the expansion of the logarithm itself includes terms with both plus and minus signs, remarkably, in all cases we get a sum over regions, with all positive integer coefficients, reflecting the allowed leading singularities for different orderings of approaching the collinear region.

\section{Some faces of the amplituhedron}

In this section, we study a few classes of lower-dimensional faces of the amplituhedron, that are particularly easy to triangulate. The canonical form associated with these faces computes corresponding cuts of the full integrand.

Ladders and next-to-ladders. Already in [1], we discussed a set of faces that are extremely easy to understand. Let us take all $L$ loops to cut the line (12), by sending all the $w_{i} \rightarrow 0$. The positivity conditions just become $\left(x_{i}-x_{j}\right)\left(z_{i}-z_{j}\right)<0$. In whatever configuration of $x$ 's we have, they are ordered in some way, say $x_{1}<\cdots<x_{L}$, and this condition tells us that the $z$ 's are oppositely ordered $z_{1}>\cdots>z_{L}$. The $y_{i}$ just have to be positive. The associated form is then trivially

$$
\frac{1}{y_{1}} \ldots \frac{1}{y_{L}} \frac{1}{x_{1}} \frac{1}{x_{2}-x_{1}} \ldots \frac{1}{x_{L}-x_{L-1}} \frac{1}{z_{L}} \frac{1}{z_{L-1}-z_{L}} \ldots \frac{1}{z_{1}-z_{2}}
$$


which corresponds to the unique "ladder" local diagrams that can contribute to this cut; to see this propagator structure explicitly, we simply regroup the terms in the product as $1 /\left(y_{1} \cdots y_{L}\right)$ multiplying

$$
\frac{1}{x_{1}} \times \frac{1}{\left(x_{2}-x_{1}\right)\left(z_{1}-z_{2}\right)} \times \cdots \times \frac{1}{\left(x_{L}-x_{L-1}\right)\left(z_{L-1}-z_{L}\right)} \times \frac{1}{z_{L}}
$$

We can move on to consider "next-to-ladder" cuts. Suppose for instance that $(L-1)$ of the loop variables cutting (12), while the $L$ 'th loop cuts (34) so that $y_{L} \rightarrow 0$. The positivity for the $(L-1)$ lines is simply $x_{1}<x_{2}<\cdots<x_{L-1}$ and $z_{1}>z_{2}>\cdots>z_{L-1}$ as above. The mutual positivity conditions are just

$$
w_{L} y_{i}>\left(x_{i}-x_{L}\right)\left(z_{i}-z_{L}\right)
$$

The canonical form is very easy to determine. We simply consider all the $L$ orderings of the $x$ 's for which $x_{1}<\cdots,<x_{L-1}$, i.e. the orderings $\left[x_{1}, \cdots, x_{L-1}, x_{L}\right]$, $\left[x_{1}, \cdots, x_{L}, x_{L-1}\right], \cdots,\left[x_{L}, x_{1}, \cdots, x_{L-1}\right] ;$ similarly, we consider all the analogous orderings of the $z$ 's: $\left[z_{L}, z_{L-1}, \cdots, z_{1}\right],\left[z_{L-1}, z_{L}, \cdots, z_{1}\right], \cdots,\left[z_{L-1}, \cdots, z_{1}, z_{L}\right]$. If in the ordering either both $x_{k}>x_{L}, z_{k}>z_{L}$ or $x_{k}<x_{L}, z_{k}<z_{L}$ we have, $y_{k}>\left(x_{k}-x_{L}\right)\left(z_{k}-z_{L}\right) / w_{L}$, otherwise we have just $y_{k}>0$. The corresponding form is

$$
\begin{gathered}
\sum_{\sigma_{1}<\cdots<\sigma_{L-1}, \rho_{1}>\cdots>\rho_{L-1}}\left[x_{\sigma_{1}^{-1}}, \cdots, x_{\sigma_{L}^{-1}}\right]\left[z_{\rho_{1}^{-1}}, \cdots, z_{\rho_{L}^{-1}}\right] \\
\times \prod_{k=1}^{L-1}\left\{\begin{array}{c}
{\left[y_{k}-\frac{1}{w_{L}}\left(x_{k}-x_{L}\right)\left(z_{k}-z_{L}\right)\right]^{-1} \sigma_{k}>\sigma_{L}, \rho_{k}>\rho_{L} \quad \text { or } \quad \sigma_{k}<\sigma_{L}, \rho_{k}<\rho_{L}} \\
y_{k}^{-1} \text { otherwise }
\end{array}\right.
\end{gathered}
$$

This expression sums the cuts for local diagrams of the form

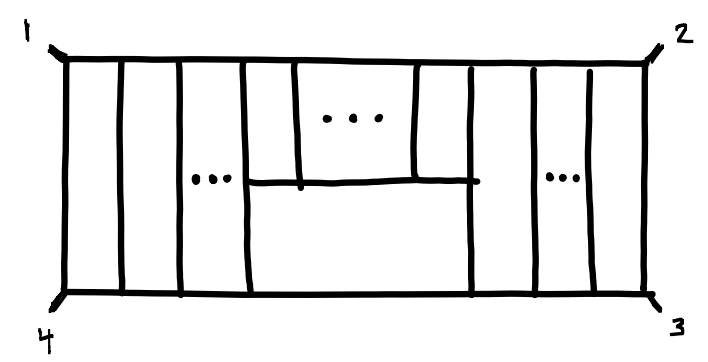

Corner cuts. We can systematically approach the faces of the amplituhedron where every line is one of the double-cut configurations. We already know what happens with the unitarity double-cut on general grounds. So we are left with the "corner cuts", where any line either passes through $Z_{i}$, or lies in the plane $\left(Z_{i-1} Z_{i} Z_{i+1}\right)$. We use different convenient gauge fixings: for the case of lines passing through 1 , and lines in the plane (412), we use

$$
D_{\text {through 1 }}=\left(\begin{array}{cccc}
1 & 0 & 0 & 0 \\
0 & y & 1 & z
\end{array}\right), \quad D_{\text {in (412) }}=\left(\begin{array}{cccc}
u & 1 & 0 & 0 \\
-v & 0 & 0 & 1
\end{array}\right)
$$

Note that

$$
\left\langle D_{\text {through } 1} D_{\text {in (412) }}\right\rangle=-1
$$


is negative, and so we immediately learn that it is impossible to have lines of both types in one corner! We can either have a collection of lines passing through 1, or a collection of lines lying in the plane (412). Suppose we approach the configuration where all the lines path through 1 , by starting with all the lines intersecting (41), and sending the lines into the corner in some order, first $w_{1} \rightarrow 0, \cdots$, then $w_{L}>0$. This orders $w_{1}<\cdots<w_{L}$ and so $y_{1}>\cdots>y_{L}$, thus the form on this final corner cut is just $\left[y_{L}, \cdots, y_{1}\right]$. Note that we see again something we have observed already a number of times: the form on the cut depends not just on the geometry of the ultimate configuration of lines, but also on the path taken to that configuration.

We can easily determine completely general corner cuts where all the lines are of one type or the other. For instance, suppose we start with $L_{1}$ lines cutting (14), and $L_{2}$ lines cutting (12), and that we send these lines to pass through the corners 1 and 2 in some order. If we parametrize the matrices as

$$
\left(\begin{array}{cccc}
1 & 0 & 0 & 0 \\
0 & y_{i} & 1 & z_{i}
\end{array}\right)\left(\begin{array}{cccc}
0 & 1 & 0 & 0 \\
-\alpha_{I} & 0 & \beta_{I} & 1
\end{array}\right)
$$

then the positivity conditions are just $y_{L_{1}}>\cdots>y_{1}, \beta_{L_{2}}>\cdots>\beta_{1}$, with the mutual positivity condition $z_{i} \beta_{I}>1$, which just means $z_{i}>1 / \beta_{1}$ for all $i$. Then the form is trivially

$$
\prod_{I} \frac{1}{\alpha_{I}} \times\left[y_{L_{1}}, \cdots, y_{1}\right]\left[\beta_{L_{2}}, \cdots, \beta_{1}\right] \prod_{i} \frac{1}{z_{i}-\beta_{1}^{-1}}
$$

This result generalizes trivially to the case with $L_{1}$ lines cutting (41), $L_{2}$ lines cutting (12), $L_{3}$ lines cutting (23) and $L_{4}$ lines cutting (34), then taken to pass through 1,2,3, 4 .
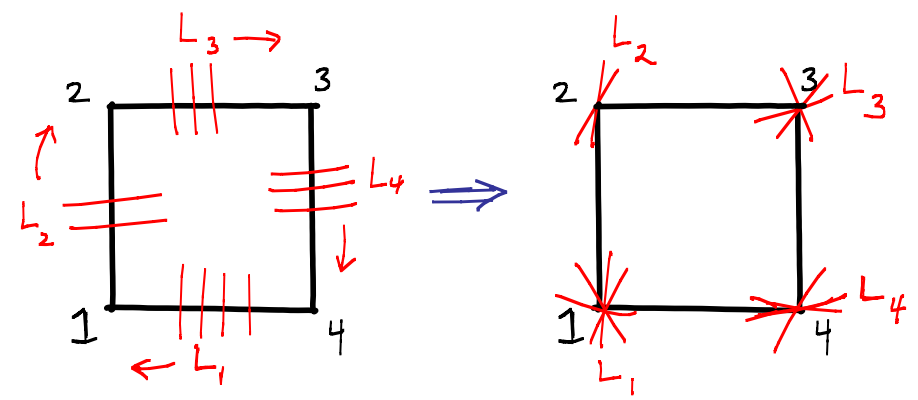

These results are very simple and arise from a single local term. Much more interesting are the mixed corner cuts, where we have the two different types of lines passing through different corners. One case is still extremely simple, where the two different lines pass through consecutive corners. Suppose we have $L_{1}$ lines passing through 1 , and $L_{2}$ lines lying in (123). It is trivial to see that

$$
\left\langle D_{\text {through } 1} D_{\text {lying in (123) }}\right\rangle=(14)_{\text {through } 1}(23)_{\text {lying in (123) }}>0
$$

and so the mutual positivity between these two sets is automatically satisfied. The form is then just the product of the form for the $L_{1}$ lines and the $L_{2}$ lines separately. 
The non-trivial case is when the corner cuts are different lines in opposite corners. Suppose we have $L_{1}$ lines cutting (41) that were then sent to pass through 1 in order $\left(L_{1}, \cdots, 1\right)$, and $L_{3}$ lines cutting (23) that were made to pass through (234) in order $\left(1, \cdots, L_{3}\right)$.

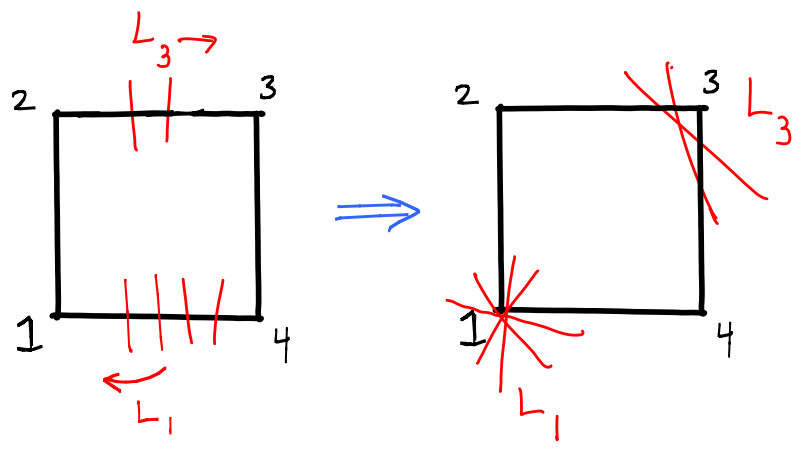

For notational convenience we'll parametrize the $D$ matrices using different variable names in this case:

$$
D_{\text {through 1 }}=\left(\begin{array}{cccc}
1 & 0 & 0 & 0 \\
0 & x_{i} & 1 & y_{i}
\end{array}\right), \quad D_{\text {lying in(234) }}=\left(\begin{array}{cccc}
0 & 1 & a_{I}^{-1} & 0 \\
0 & 0 & b_{I}^{-1} & 1
\end{array}\right)
$$

Then the positivity conditions are

$$
x_{1}<\cdots<x_{L_{1}}, a_{1}<\cdots<a_{L_{3}} \text {, and } \frac{x_{i}}{a_{I}}+\frac{y_{i}}{b_{I}}>1
$$

It is quite straightforward to triangulate this space; let us work out the case $L_{3}=2$ explicitly. Here the geometry is very similar to the last of our warmup exercises. Suppose first that $b_{1}<b_{2}$. Then the inequalities are just $x_{i} / a_{2}+y_{i} / b_{2}>1$ together with the restriction $x_{1}<\cdots<x_{L_{1}}$. We simply order the $x_{i}$ relative to $a_{2}$. If $x_{i}>a_{2}$, then we just have $y_{i}>0$ and the form is $1 / y_{i}$, while if $x_{i}<a_{2}$, we have $Y_{i, 2}>0$ and the form is $1 / Y_{i, 2}$. Here we have defined

$$
Y_{i, 1}=y_{i}+\frac{b_{1} x_{i}}{a_{1}}-b_{1}, Y_{i, 2}=y_{i}+\frac{b_{2} x_{i}}{a_{2}}-b_{2}
$$

Thus, for $b_{1}<b_{2}$, the form is just

$$
\frac{1}{a_{1}\left(a_{2}-a_{1}\right)} \frac{1}{b_{1}\left(b_{2}-b_{1}\right)} \sum_{m}\left[\cdots, x_{m}, \underline{a_{2}}, x_{m+1} \cdots\right] \prod_{k}\left\{\begin{array}{cc}
Y_{k, 2}^{-1} & k \leq m \\
y_{k}^{-1} & k>m
\end{array}\right\}
$$

If instead $b_{2}<b_{1}$, then we have to break $x$ space up into the three regions between $0, a_{12}, a_{2}$ where $a_{12}=\frac{a_{1} a_{2}\left(b_{1}-b_{2}\right)}{a_{2} b_{1}-a_{1} b_{2}}$. We have to sum over all the orderings of the $x$ 's relative to $a_{12}, a_{2}$ '; for all the $x_{i}>a_{2}$, the form in $y$ space is just $1 / y_{i}$, for the $x_{i}$ in the range $a_{2}>x_{i}>a_{12}$ the $y$ form is just $1 / Y_{i, 2}$, while for $a_{12}>x_{i}>0$ the $y$ form is $1 / Y_{i, 1}$. Thus in this case the form is

$$
\frac{1}{a_{1}\left(a_{2}-a_{1}\right)} \frac{1}{b_{1}\left(b_{2}-b_{1}\right)} \sum_{m \leq l}\left[\cdots, x_{m}, \underline{a_{12}}, x_{m+1}, \cdots, x_{l}, \underline{a_{2}}, x_{l+1}, \cdots\right] \prod_{k}\left\{\begin{array}{c}
Y_{k, 1}^{-1}, k \leq m \\
Y_{k, 2}^{-1}, m<k \leq l \\
y_{k}^{-1} k>l
\end{array}\right\}
$$


The full form is just the sum of these two pieces. While this result is completely straightforward from triangulation, it gives rise to highly non-trival local expressions even at comparatively low loop order. In the first really interesting case at 5 loops, with $L_{1}=3$ and $L_{3}=2,19$ local terms contribute to this cut, and when they are all combined under a common denominator, the numerator has 325 terms.

There is another interesting feature of these cuts, which is not evident from any traditional point of view but is obvious from the positive geometry. We have seen that fixing the order in which the lines are brought to pass through 1, imposes the constraint $x_{1}<\cdots<x_{L_{1}}$. However, if we sum over all the different orderings, we simply remove these ordering constraints! We then expect that the form simplifies greatly. Indeed, if we stick to the case $L_{3}=2$, then we just get several copies of the problem $x / a_{1}+y / b_{1}>1, x / a_{2}+y / b_{2}>1$, which we analyzed in our warmup section. Thus, the sum over all the ways to start with $L_{1}$ lines on (41) which are then sent through 1 (while sending $L_{3}=2$ lines to lie in (234) in the usual fixed order), is

$$
\begin{aligned}
\frac{1}{a_{1}\left(a_{2}-a_{1}\right)}\left[\frac { 1 } { b _ { 1 } ( b _ { 2 } - b _ { 1 } ) } \prod _ { i } \left(\left[x_{i}, \underline{\left.a_{2}\right]} \frac{1}{Y_{i, 2}}+\left[\underline{a_{2}}, x_{i}\right] \frac{1}{y_{i}}\right)\right.\right. \\
+\frac{1}{b_{2}\left(b_{1}-b_{2}\right)} \prod_{i}\left(\left[x_{i}, \underline{\left.a_{12}\right]} \frac{1}{Y_{i, 1}}+\left[\underline{a_{12}}, x_{i}, \underline{a_{2}}\right] \frac{1}{Y_{i, 2}}+\left[\underline{a_{2}}, x_{i}\right] \frac{1}{y_{i}}\right)\right]
\end{aligned}
$$

Internal cuts. It is interesting that up to 4 loop order, every loop in the local expansion of the amplitude touches the external lines, but this behavior is obviously not generic. Starting at 5 loops, we have diagrams with purely internal loops, such as

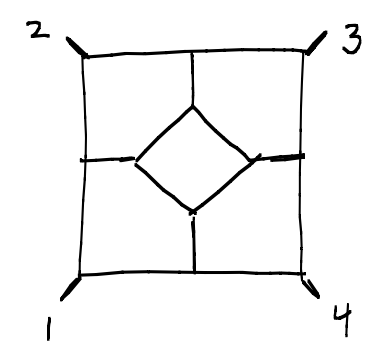

and it is interesting to probe these from positivity. Let us look at a particularly simple set of cuts that exposes the structure in a nice way. Suppose we take 4 lines $(A B)_{1}, \cdots,(A B)_{4}$, and take them to pass through $1,2,3,4$ respectively. But additionally, we take the cut where $\left\langle A B_{1} A B_{2}\right\rangle \rightarrow 0,\left\langle A B_{2} A B_{3}\right\rangle \rightarrow 0,\left\langle A B_{3} A B_{4}\right\rangle \rightarrow 0,\left\langle A B_{4} A B_{1}\right\rangle \rightarrow 0$, i.e. the lines are taken to one intersect the next. The $D$ matrices are simply

$$
\begin{aligned}
D_{(1)} & =\left(\begin{array}{cccc}
1 & 0 & 0 & 0 \\
0 & \alpha^{-1} & 1 & \beta
\end{array}\right), & D_{(2)} & =\left(\begin{array}{cccc}
0 & 1 & 0 & 0 \\
\gamma & 0 & \beta^{-1} & 1
\end{array}\right) \\
D_{(3)} & =\left(\begin{array}{cccc}
0 & 0 & 1 & 0 \\
-1 & -\sigma & 0 & \gamma^{-1}
\end{array}\right), & D_{(4)} & =\left(\begin{array}{cccc}
0 & 0 & 0 & 1 \\
-\sigma^{-1} & -1 & -\alpha & 0
\end{array}\right)
\end{aligned}
$$


Note that the mutual positivity between $D_{(1)} D_{(3)}$ and $D_{(2)} D_{(4)}$ is automatic. The geometry of the lines is

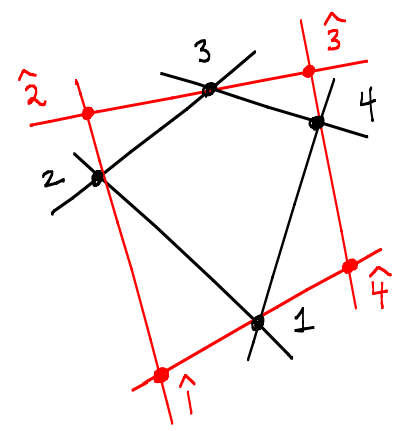

and so we can think of the lines as $(A B)_{1}=\hat{1} \hat{2},(A B)_{2}=\hat{2} \hat{3},(A B)_{3}=\hat{3} \hat{4},(A B)_{4}=\hat{1} \hat{4}$, where

$$
\begin{array}{ll}
\hat{1}=1+\sigma(2+\alpha(3+\beta 4)), & \hat{2}=2+\alpha(3+\beta(4-\gamma 1)) \\
\hat{3}=3+\beta(4-\gamma(1+\sigma 2)), & \hat{4}=4-\gamma(1+\sigma(2+\alpha 3))
\end{array}
$$

Now, it is easy to see that the remaining mutual positivity conditions between $D_{(1)}, \cdots, D_{(4)}$ and the other $D_{(i)}$ are just satisfied by the lower-loop shifted amplitude; thus we conclude that on this cut the form is

$$
\frac{d \alpha}{\alpha} \frac{d \beta}{\beta} \frac{d \gamma}{\gamma} \frac{d \sigma}{\sigma} \times M^{L-4}(\hat{1}, \hat{2}, \hat{3}, \hat{4})
$$

\section{Four particle outlook}

We have only scratched the surface of the rich amplituhedron geometry controlling four-particle scattering in planar $\mathcal{N}=4 \mathrm{SYM}$ at all-loop order. There is obviously much more to be done just along the elementary lines of this note, minimally in further continuing a systematic exploration of other facets of the geometry, corresponding to different classes of cuts of physical interest. But we close with a few comments about some different avenues of exploration.

In this note we have approached the determination of the integrand for four-particle scattering by directly "triangulating" the amplituhedron geometry. The $L-$ loop geometry is defined in a self-contained way, as a subspace living inside $L$ copies of space-time realized as $G(2,4)$. In particular, nowhere do we need to refer to lower-loop, higher- $k$ amplitudes, as in necessary in the BCFW recursion approach [15] to loop integrands [12]. Nonetheless, it is likely that some natural connection exists with the full problem, and perhaps a broader view of the bigger amplituhedron geometry in which the four-particle problem sits will be important for systematically determining the all-loop integrand. Certainly, experience with the positive Grassmannian $[2,4,5]$ strongly suggests that different faces can't be properly understood in isolation.

As we have seen, the approach to computing the integrand by triangulating the amplituhedron does not give us the familiar expansions that are manifestly local. This is of 
course not surprising; however, what is surprising is that some special local expansions expose yet another aspect of positivity, that we are not making apparent in the triangulation approach. As also mentioned in [1], we are still clearly missing a picture of the form $\Omega$ which is analogous to one available for convex polygons, determined by a literal volume of the dual polygon. We don't yet have a notion of a "dual amplituhedron", but there is a powerful indication that such a formulation must exist: the form $\Omega$ is itself positive, inside the amplituhedron! More specifically, we can write the $L$-loop integrand as

$$
\Omega_{L}\left(A B_{i}\right)=\prod_{i=1}^{L}\left\langle A B_{i} d^{2} A_{i}\right\rangle\left\langle A B_{i} d^{2} B_{i}\right\rangle M_{L}\left(A B_{i}\right)
$$

Then, we claim that when the $(A B)_{i}$ are taken to lie inside the amplituhedron,

$$
M_{L}\left(A B_{i}\right)>0
$$

We will return to exploring this fact at greater in length in [16]. We stress that this property is not manifest term-by-term in the amplituhedron triangulation expansion of the integrand. Random forms of the local expansion also don't make this remarkable property manifest term-by-term, but there are particularly nice forms of local expansion that do make this manifest. As we will discuss in [16], we suspect that this surprising positivity property of the integrand is pointing the way to a more direct and intrinsic, triangulationindependent definition for the canonical form $\Omega$ associated with the amplituhedron.

From a mathematical point of view, it is interesting that the study of amplitudes leads to stratifications of various collections of objects in projective space. If we consider a collection of $n$ vectors in $k$ dimensions, together with a cyclic structure on this data, we are led to the beautiful stratification of the space given by the positive Grassmannian. Even just with the the four-particle amplituhedron, we see something new, not needing a cyclic structure on the objects: given a collection of $L$ 2-planes in 4 dimensions, the positivity conditions are fully permutation invariant between the $L$ lines. Just as with the positive Grassmannian, it is natural to expect the cell structure of the amplituhedron to be determined in a fundamentally combinatorial way. The fascinating path-dependence of the forms associated with the cuts, together with the combinatorics that arise just in the simple discussion of the multi-collinear limit, are perhaps indications of an underlying combinatorial structure.

The four-particle amplitude is a truly remarkable object. At the level of the integrand, at multi-loop order it contains non-trivial information about all the more complicated multiparticle amplitudes in the theory. At the level of the final integrated expression, we have a function that smoothly interpolates between a picture of "interacting gluons" at weak coupling to "minimal area surface in AdS space" [17] at strong coupling. We have explored a reformulation of this physics in terms of a simple to define, yet rich and intricate geometry. We hope that this will lead us to a more direct understanding of how the picture of "gluons" and "strings" arise as different limits of a single object. As a small step in this direction, it is encouraging to find a natural understanding, intrinsic to the geometry, of the behavior of the amplitude in the multi-collinear region, and an associated intrinsic-to-the-geometry rationale for taking the log of the amplitude. Trying to more completely determine the IR 
singular behavior of the integrand of the amplitude is an ideal laboratory to connect our approach to the loop integrand with the final integrated expressions, and especially to ideas related to integrability. Indeed the coefficient of the $\log ^{2}$ infrared divergence of the log of the amplitude is given by the cusp anomalous dimension, which was brilliantly determined using integrability in [18-20]. It is notable that this approach makes crucial use of a spectral parameter, something which is absent in our present discussion of the amplituhedron. Given the spectral deformation of on-shell diagrams given in [21,22], it is natural to ask whether a similar deformation can be found directly at the level of the amplituhedron.

\section{Acknowledgments}

We thank Jake Bourjaily, Freddy Cachazo, Simon Caron-Huot, Johannes Henn, Andrew Hodges, Jan Plefka, Dave Skinner and Matthias Staudacher for stimulating discussions. N. A.-H. is supported by the Department of Energy under grant number DE-FG0291ER40654. J. T. is supported in part by the David and Ellen Lee Postdoctoral Scholarship and by DOE grant DE-FG03-92-ER40701 and also by NSF grant PHY-0756966.

Open Access. This article is distributed under the terms of the Creative Commons Attribution License (CC-BY 4.0), which permits any use, distribution and reproduction in any medium, provided the original author(s) and source are credited.

\section{References}

[1] N. Arkani-Hamed and J. Trnka, The amplituhedron, JHEP 10 (2014) 030 [arXiv: 1312.2007] [INSPIRE].

[2] A. Postnikov, Total positivity, Grassmannians and networks, math/0609764 [INSPIRE].

[3] V.V. Fock and A.B. Goncharov, Cluster ensembles, quantization and the dilogarithm, math/0311245 [INSPIRE].

[4] N. Arkani-Hamed, F. Cachazo, C. Cheung and J. Kaplan, A duality for the S matrix, JHEP 03 (2010) 020 [arXiv: 0907.5418] [inSPIRE].

[5] N. Arkani-Hamed et al., Scattering amplitudes and the positive Grassmannian, arXiv: 1212.5605 [INSPIRE].

[6] A. Hodges, Eliminating spurious poles from gauge-theoretic amplitudes, JHEP 05 (2013) 135 [arXiv: 0905.1473] [INSPIRE].

[7] Z. Bern, M. Czakon, L.J. Dixon, D.A. Kosower and V.A. Smirnov, The four-loop planar amplitude and cusp anomalous dimension in maximally supersymmetric Yang-Mills theory, Phys. Rev. D 75 (2007) 085010 [hep-th/0610248] [INSPIRE].

[8] Z. Bern, L.J. Dixon and V.A. Smirnov, Iteration of planar amplitudes in maximally supersymmetric Yang-Mills theory at three loops and beyond, Phys. Rev. D 72 (2005) 085001 [hep-th/0505205] [INSPIRE].

[9] Z. Bern, J.J.M. Carrasco, H. Johansson and D.A. Kosower, Maximally supersymmetric planar Yang-Mills amplitudes at five loops, Phys. Rev. D 76 (2007) 125020 [arXiv:0705.1864] [INSPIRE]. 
[10] J.L. Bourjaily, A. DiRe, A. Shaikh, M. Spradlin and A. Volovich, The soft-collinear bootstrap: $N=4$ Yang-Mills amplitudes at six and seven loops, JHEP 03 (2012) 032 [arXiv: 1112.6432] [INSPIRE].

[11] B. Eden, P. Heslop, G.P. Korchemsky and E. Sokatchev, Constructing the correlation function of four stress-tensor multiplets and the four-particle amplitude in $N=4$ SYM, Nucl. Phys. B 862 (2012) 450 [arXiv:1201.5329] [INSPIRE].

[12] N. Arkani-Hamed, J.L. Bourjaily, F. Cachazo, S. Caron-Huot and J. Trnka, The all-loop integrand for scattering amplitudes in planar $N=4$ SYM, JHEP 01 (2011) 041 [arXiv: 1008.2958] [INSPIRE].

[13] S. Caron-Huot, Loops and trees, JHEP 05 (2011) 080 [arXiv: 1007.3224] [INSPIRE].

[14] N. Arkani-Hamed, J.L. Bourjaily, F. Cachazo and J. Trnka, Local integrals for planar scattering amplitudes, JHEP 06 (2012) 125 [arXiv: 1012.6032] [INSPIRE].

[15] R. Britto, F. Cachazo, B. Feng and E. Witten, Direct proof of tree-level recursion relation in Yang-Mills theory, Phys. Rev. Lett. 94 (2005) 181602 [hep-th/0501052] [INSPIRE].

[16] N. Arkani-Hamed, A. Hodges and J. Trnka, Positive amplitudes in the amplituhedron, arXiv: 1412.8478 [INSPIRE].

[17] L.F. Alday and J.M. Maldacena, Gluon scattering amplitudes at strong coupling, JHEP 06 (2007) 064 [arXiv: 0705.0303] [INSPIRE].

[18] N. Beisert and M. Staudacher, The N=4 SYM integrable super spin chain, Nucl. Phys. B 670 (2003) 439 [hep-th/0307042] [INSPIRE].

[19] N. Beisert, B. Eden and M. Staudacher, Transcendentality and crossing, J. Stat. Mech. 01 (2007) P01021 [hep-th/0610251] [INSPIRE].

[20] B. Eden and M. Staudacher, Integrability and transcendentality, J. Stat. Mech. 11 (2006) P11014 [hep-th/0603157] [INSPIRE].

[21] L. Ferro, T. Lukowski, C. Meneghelli, J. Plefka and M. Staudacher, Spectral parameters for scattering amplitudes in $N=4$ super Yang-Mills theory, JHEP 01 (2014) 094 [arXiv: 1308.3494] [INSPIRE].

[22] L. Ferro, T. Łukowski, C. Meneghelli, J. Plefka and M. Staudacher, Harmonic R-matrices for scattering amplitudes and spectral regularization, Phys. Rev. Lett. 110 (2013) 121602 [arXiv: 1212.0850] [INSPIRE]. 